\title{
Ubiquitination: Friend and Foe in Cancer
}

Mohammed A. Mansour 1,2,3

${ }_{1}$ Institute of Cancer Sciences, University of Glasgow and 2 The CRUK Beatson

Institute, Glasgow, Switchback Road, G61 1BD, United Kingdom.

${ }_{3}$ Biochemistry Division, Department of Chemistry, Faculty of Science, Tanta

University, Tanta, 31527, Egypt.

\section{Address correspondence to:}

Mohammed A. Mansour, Ph.D.: Institute of Cancer Sciences, University of Glasgow, Garscube Estate, Switchback Road, Glasgow, G61 1QH, UK.

Phone: +44 (0) 754-380-5794

E-mails: mohammed.mansour@glasgow.ac.uk

biomansour@hotmail.com

Declarations of interest: none

Keywords: ubiquitin-proteasome system; cancer; E3 ligases; MDM2; P53; SCF complex; deubiquitinating enzyme; PROTAC; hydrophobic tag

Abbreviations: Ub, ubiquitin; UBLs, ubiquitin-like proteins; DUBs, deubiquitinating enzymes; TCR, T cell receptor; UPS, ubiquitin-proteasome system; IAPs, inhibitors of apoptosis-related proteins; GAPs, GTPase-activating proteins; PI3K, phosphatidylinositol 3-kinase; HECT, homology to E6AP C-terminus; RING, really interesting new gene; CDK, cyclin-dependent kinase; USP, ubiquitin-specific protease; PROTACs, protein-targeting chimeric molecules; HyT, hydrophobicity tags; NF-kB, nuclear factor kappa-B; TCGA, The Cancer Genome Atlas; UCP, ubiquitin carrier protein; MMP, matrix metalloprotease. 


\begin{abstract}
Dynamic modulation and posttranslational modification of proteins are tightly controlled biological processes that occur in response to physiological cues. One such dynamic modulation is ubiquitination, which marks proteins for degradation via the proteasome, altering their localization, affecting their activity, and promoting or interfering with protein interactions. Hence, ubiquitination is crucial for a plethora of physiological processes, including cell survival, differentiation and innate and adaptive immunity. Similar to kinases, components of the ubiquitination system are often deregulated, leading to a variety of diseases, such as cancer and neurodegenerative disorders. In a context-dependent manner, ubiquitination can regulate both tumor-suppressing and tumor-promoting pathways in cancer. This review outlines how components of the ubiquitination systems (e.g. E3 ligases and deubiquitinases) act as oncogenes or tumor suppressors according to the nature of their substrates. Furthermore, I interrogate how the current knowledge of the differential roles of ubiquitination in cancer lead to technical advances to inhibit or reactivate the components of the ubiquitination system accordingly.
\end{abstract}




\section{Introduction}

Ubiquitination is a posttranslational modification of proteins in both normal homeostasis and disease. This process involves the addition of an evolutionarily conserved small protein, ubiquitin (Ub) or ubiquitin-like proteins (UBLs), to target proteins for proteasome degradation or non-degradative signaling (1). The Ub signal on modified proteins is covalently coupled to lysine side-chain residues in a sequential manner by a cascade of enzymatic reactions involving collaboration between the activating (E1), conjugating (E2) and ligating (E3) enzymes (2). The Cterminus of $\mathrm{Ub}$ is first activated by an E1 enzyme and is then transferred onto the active site cysteine of an E2 conjugating enzyme through trans-thioesterification. Subsequently, E3 Ub ligases (HECT (Homology to E6AP C Terminus) or RING (Really Interesting New Gene)) bind simultaneously the E2-Ub intermediate and the target protein to catalyze isopeptide bond formation between the C-terminal glycine of ubiquitin and the substrate lysine residue (3) (Fig. 1). The human genome contains around 50 genes encoding E2 enzymes and 600 genes encoding E3 ligases. Also, there are more than 90 deubiquitinating enzymes (DUBs), which can remove Ub from the Ub-bound proteins. DUBs can be divided into six classes: ubiquitin C-terminal hydrolases (UCHs), ubiquitin-specific proteases (USPs), ovarian-tumor proteases (OTUs), JAMM/MPN domain-associated metallopeptidases (JAMMs), MachadoJoseph disease protein domain proteases (MJD) and monocyte chemotactic proteininduced protein (MCPIP) $(4,5)$.

The mono-Ub proteins undergo multiple ubiquitination reactions to generate multi mono-Ub proteins or polymeric Ub chains. Both mono-Ub and multi mono-Ub are involved in several biological processes, including endocytosis, DNA repair, and protein localization and trafficking (6). Because ubiquitin itself has seven lysine (K) residues, this modification can be propagated, by transferring additional ubiquitin to one of seven lysine residues or the N-terminus $-\mathrm{NH} 2$ group (7). According to the formed chain topology, ubiquitination can have different biological outcomes. For instance, K48 and K11 chains are related to degradation by the proteasome, whereas K63 and linear ubiquitin chains have a scaffolding role for signaling assemblies and play a prominent role in many biological pathways, including inflammation. However, K6 and K27 poly-ubiquitinated proteins are associated with DNA damage responses and mitochondrial maintenance, respectively. Also, K29 and K33 polyubiquitinated proteins are related to lysosomal degradation and T cell receptor (TCR) signaling, respectively (8-10) (Fig. 2).

Ubiquitination regulates a variety of complex cellular processes, including protein degradation, protein-protein interactions, endocytosis, cell cycle progression, and activating or inactivating substrates (9). Therefore, any functional mutation or aberrant expression of the $\mathrm{Ub}$ system components can lead to several disorders, including cancer, neurodegenerative disorders, and adaptive and innate immunityrelated disorders. The physiological functions of ubiquitination are not limited to proteolysis. There are also nonproteolytic roles of ubiquitination such as multiprotein complex assembly, inflammatory signaling, autophagy, DNA repair and regulation of enzymatic activity (11-13).

In cancer, ubiquitination may lead to the activation or deactivation of tumorigenic pathways. Several reports have shown that aberrant expression of E3 ligases and DUBs are associated with human malignancies by regulating the activity or degradation of tumor-promoting or -suppressor proteins. Prominent examples include cyclin-dependent kinase inhibitor 1B (p27), p53 and nuclear factor kappa-B (NF-kB) (14-16). Unlike kinases, most components of the ubiquitin system do not have well-defined catalytic pockets and require a dynamic rearrangement of multiple protein-protein interactions, making them very difficult for inhibition by small 
molecules. However, with advances in technologies and better understanding of ubiquitin biology, there have been great developments in the reactivation of the ubiquitination system using cutting-edge methodologies. For instance, proteintargeting chimeric molecules (PROTACs) and hydrophobicity tags (HyT) have been developed to modulate the ubiquitination system and the fate of modified proteins (17). In this review, I will discuss how genetic defects in components of the Ub system can mediate progression or suppression of the tumorigenic pathways in different types of cancer. I will also shed light on the current and future perspectives of cancer therapeutics that depend on either activation or deactivation of the ubiquitination of target proteins (Fig. 3).

\section{Molecular mechanisms of tumor-promoting roles of Ub system components}

\subsection{Tumor-promoting E3 ligases}

\subsubsection{E3 ligases as degraders of tumor suppressing proteins}

\section{MDM2/p53 interaction}

One of the well-known functions of ubiquitination is the modulation of protein stability through the ubiquitin-proteasome system (UPS) in normal and pathological states. Proteins that are marked by Ub are trafficked to the proteasome or lysosome for degradation (Fig. 1). Mutations or deregulation of the expression of key players in this process, E3 ligases, are found in different carcinomas and usually correlate clinically with poor survival and prognosis (18-20). For instance, the guardian of the genome, p53, controls the regulation and expression of cell cycle arrest, DNA repair and apoptosis genes in normal homeostasis (21). However, in cancer cells, p53 undergoes ubiquitination upon the binding of the RING finger E3 ubiquitin-protein ligase MDM2. MDM2 interacts with the p53 N-terminus transactivation domain (TAC), leading to ubiquitination and subsequent degradation by the ubiquitinproteasome system (22,23) (Fig. 4). Hence, MDM2 is overexpressed in different types of cancer and negatively correlates with p53 protein levels, resulting in poor survival and prognosis $(24,25)$. Analysis of MDM2 genetic aberrations in cancers by The Cancer Genome Atlas (TCGA) cBioportal revealed multiple genetic alterations. Mutation and amplification are the two most predominant alterations found in several carcinomas, including sarcoma, urogenital, breast and brain malignancies (Fig. 5). Gain-of-function mutations in, and amplification of, MDM2 in several malignancies are suggested to increase MDM2 activity levels and subsequent degradation of p53. Moreover, a recently identified E3 ligase targeting p53 for degradation in cancer is the E3 ligase RING1 (26). RING1 directly interacts with and ubiquitinates p53, resulting in its proteasomal degradation. The RING domain of RING1 is required for its Ub ligase activity. Consequently, knocking RING1 down inhibits cancer cell proliferation and survival, and induces cell cycle arrest, apoptosis and senescence. Clinically, RING1 expression is upregulated in cancer cells and associates with poor prognosis $(26,27)$.

\section{SCFSKP2 complex}

Another exciting example is the ubiquitination protein complex SCFSKP2. This complex consists of the F-box proteins SKP2, CUL1 and SKP1 and the RING finger protein RBX1. SKP2 from this complex has shown an oncogenic potential clinically by its overexpression in several malignancies and its inverse correlation with critical cyclin-dependent kinase (CDK) inhibitors, including p27KIP1 (28-30). Analysis by TCGA showed several types of mutations and amplifications of SKP2 in different types of cancers, including cervical, endometrial, adrenocortical, ovarian, breast and non-small cell lung cancers (Fig. 6). These gain-of-function mutations and amplifications of SKP2 are thought to cause upregulation of SKP2 expression to 
promote its oncogenic functions. Mechanistically, SKP2 has an oncogenic function via ubiquitination of CDK inhibitors (p27KIP1 and p21CIP1), p57 (31), p130 (32) and FOXO1 (33). Also, SKP2 plays a crucial role in AKT ubiquitination and membrane recruitment (34). Hence, due to the clinical relevance of SKP2, several attempts have been made to inhibit this complex and most importantly its interaction with either p27 or SKP1 (35-37).

Upstream regulators of SKP2 have been extensively studied to target SKP2 oncogenic function in different cancers. For example, the sequestosome 1 (SQSTM1/p62) stabilizes SKP2 in esophageal squamous cell carcinoma (ESCC) and enhances cell apoptosis resistance and tumor growth (38). Consistently, immunohistochemical analysis of primary ESCC tissues revealed a positive correlation between p62 and SKP2. These findings propose that p62 is an early biomarker and a therapeutic target in ESCC (38). Recently, Su et al. (39) suggest that miR-3163 and the maternally expressed gene 3 (Meg3) may coordinate suppression of translation of Skp2 mRNA in non-small-cell lung carcinoma (NSCLC) cells to inhibit cancer cell growth. Moreover, in neuroblastoma cells, a positive correlation was identified between MYCN activity and SKP2 mRNA levels. MYCN was found to bind directly to E-boxes within the SKP2 promoter since SKP2 transcriptional activity was decreased by either the removal of MYCN or E-box mutation (40).

Downstream targets of SKP2 have also been investigated to find potential candidates as therapeutic targets. Recently, Wang et al. (41) reported that MutT homolog 1 (MTH1) is regulated by polyubiquitination mediated by the E3 ligase Skp2 in melanoma cells. It has been suggested that MTH1 is upregulated commonly mainly due to its improved stability caused by K63-linked polyubiquitination. MTH1 helps prevent misincorporation of damaged dNTPs into genomic DNA to help cancer cells evade apoptosis or DNA damage (42). A positive correlation of Skp2 and MTH1 expression was observed in melanoma cell lines and patient specimens (41). These findings identify Skp2-mediated K63-linked polyubiquitination of MTH1 as a potential candidate therapeutic target to improve melanoma treatment. In addition, Lu et al. (43) found that depletion of SKP2 decreases the enhancer of zeste homolog 2 (EZH2) levels in prostate cancer cells through upregulation of TRAF6-mediated and K63-linked ubiquitination of EZH2 for proteasomal degradation. EZH2 is highly associated with aggressive features and activation of progenitor genes and androgen receptor (AR)-target genes in prostate cancer (44). Skp2, EZH2 and histone H3 lysine 27 trimethylation (H3K27me3) are upregulated in both Pten null mouse embryonic fibroblasts (MEFs) and Pten null mouse prostate tissues (43). These findings present an important signaling network of SKP2-TRAF6-EZH2/H3K27me3 as a therapeutic target in prostate cancer.

\section{Inhibitors of apoptosis-related proteins (IAPs)}

Inhibitors of apoptosis-related proteins (IAPs) constitute a class of ubiquitination proteins responsible for binding and degradation of apoptotic caspases (45). Most IAPs contain a RING domain at the C-terminus required for ubiquitination of their substrates and auto-ubiquitination of other IAPs, including c-IAP1, c-IAP2 and Xlinked inhibitor of apoptosis (XIAP) $(46,47)$. IAPs are clinically associated with evasion of apoptosis in multiple myelomas. c-IAP1 and c-IAP2 are also linked to noncanonical NF- $\mathrm{kB}$ signaling since they promote ubiquitination and proteasomal degradation of NF-kB-inducing kinase (NIK) (48). BIRC2 and BIRC3 (encoding cIAP1 and c-IAP2, respectively) are amplified and linked to the transcription factor and oncogene YAP in several malignancies, including hepatocarcinoma, melanoma, medulloblastoma, as well as in pancreatic, lung, oral squamous cell and oesophageal 
cancers (49). Future efforts are needed to target these proteins using small molecule inhibitors or antisense oligonucleotides.

\subsubsection{E3 ligases as regulators of oncogenic activity}

The effects of ubiquitination on proteins are diverse, including proteasomal degradation, localization and activity modulation (Fig. 2). Regarding activity modulation, ubiquitination is known to regulate the activity of both oncogenes and tumor suppressors in cancer. For example, the oncogenic GTPase K-RAS was previously shown to be ubiquitinated by Rabex -5 to promote its endosomal localization by beta-TrCP (TrCP), a key member of the SkpI-Cdc53-F-box E3, to mediate its proteasomal degradation (50). However, ubiquitination also impairs KRas response to GTPase-activating proteins (GAPs). Therefore, the amount of active $\mathrm{K}$-Ras (GTP-bound) and its binding to downstream effectors are enhanced even in the absence of receptor stimuli (51). Moreover, the K-Ras G12V mutation is found in colorectal, lung and pancreatic carcinomas (52). This mutation allows K-Ras ubiquitination to enhance its binding to phosphatidylinositol 3-kinase (PI3K), thus activating PI3K-protein kinase B (AKT) signaling (6). The PI3K-AKT pathway is a signal transduction pathway that promotes cancer cell survival and growth in response to extracellular signals, hence, $\mathrm{K}$-Ras ubiquitination is a potential target to block AKT signaling in cancer (53).

\subsubsection{Metastasis-promoting E3 ligases}

Cancer cells leave the primary sites and metastasize at distant sites by disruption of cell-to-cell adhesion and promotion of epithelial-mesenchymal transition (EMT). EMT is promoted by several transcription factors and membrane proteins deregulated in cancer cells (54-60). TIAM1 is a guanine nucleotide exchange factor, at cell-to-cell junctions, which is critical for maintaining cell-to-cell contact. TIAM1 expression is modulated by HECT UBA and WWE domain-containing protein 1 (HUWE1) E3 ubiquitin ligase (61). An inverse correlation between HUWE1 and TIAM1 protein levels is observed in squamous cell lung carcinoma tissue samples. Therefore, knockdown or depletion of HUWE1 would be of great importance to stabilize TIAM1-mediated cell-to-cell junctions and stop metastasis (62). Another interesting example is the E3 ligase activity of GP78, which modulates the expression of KAI1 in sarcoma metastasis. Downregulation of GP78 by inhibitors accumulates KAI1 and results in apoptosis and reduction of the metastatic potential of sarcoma cells (63).

\subsubsection{Tumor-promoting activators of E3 ligases}

Activators of E3 ligases can also confer the tumor-promoting functions of E3 ligases. For instance, the RNA helicase, DHX15, regulates androgen receptor (AR) activity in prostate cancer cells by modulating E3 ligase Siah2-mediated AR ubiquitination (64). Consequently, DHX15-mediated AR activation is critical for prostate cancer progression, including castration-resistant prostate cancer. In addition, DHX15 expression is upregulated in prostate cancer specimens and correlates with Gleason scores and prostate-specific antigen (PSA) recurrence (64). Another example is the RING domain-containing ubiquitin E3 ligase MARCH7. As described previously, p53 activity is mainly regulated by the ubiquitin E3 ligase Mdm2, which targets p53 for ubiquitin-dependent degradation. Interestingly, it has recently been reported that MDM2 is regulated by MARCH7. MARCH7 activates K63-linked polyubiquitination of MDM2, which impedes its autoubiquitination and proteasomal degradation. Hence, MARCH7 regulates cancer cell proliferation and apoptosis in a p53dependent manner (65). 


\subsection{Tumor-promoting E2 enzymes}

The E2 conjugating enzymes are also mediating critical functions in cancer cells. For example, the ubiquitin-conjugating enzyme E2 L3 (UBE2L3) is overexpressed in NSCLC tissues as compared to normal tissue. Overexpression of UBE2L3 correlates with advanced tumor stage and poor prognosis (66). Mechanistically, Ma et al. (66) demonstrated that UBE2L3 interacts with Skp2, thereby promoting the ubiquitination and proteasomal degradation of p27kip1. Consequently, depletion of UBE2L3 expression inhibits NSCLC progression, while exogenous expression of UBE2L3 promotes NSCLC cell growth in a cell cycle-dependent manner (66). Therefore, UBE2L3 is proposed as a novel biomarker for prognosis and a potential therapeutic target for NSCLC patients.

\subsection{Tumor-promoting deubiquitinating enzymes}

Deubiquitinases function as tumor-promoting proteins if their substrates act as promoters of cancer progression. For example, USP1 and DUB3 preserve the characteristics of mesenchymal stem cells by deubiquitinating inhibitors of DNAbinding proteins in osteosarcoma (67). They can also stabilize CDC25 to activate cell cycle progression (68). USP1 is also implicated in Fanconi leukemia, in which it deubiquitinates two critical DNA repair proteins, FANCD2 and PCNA (69). Moreover, USP4 enhances the oncogenic transforming growth factor- $B$ (TGF- $B$ ) signaling and is overexpressed in invasive breast cancer. USP4 deubiquitinates activated ALK5 leading to stabilization of downstream activation of SMAD2 and SMAD2/SMAD4 complex formation (70). Importantly, activation of TGF- $B$ signaling induces expression of metastasis-related genes, such as IL-11, CXCR4 and MMPs, in breast cancer (71). Unlike previous USPs, USP7 modulates the localization and conformational structure editing of the well-known tumor suppressors p53 and PTEN. Deubiquitination of PTEN by USP7 inactivates PTEN by nuclear exclusion, leading to impairment of tumor growth (72). Consequently, USP7 is frequently overexpressed in prostate and non-small cell lung carcinomas, as well as acute promyelocytic leukemia (61).

\section{Therapeutic inhibition of tumor-promoting ubiquitination in cancer}

\subsection{Targeting the proteasome}

There are currently two proteasome inhibitors approved by the FDA: bortezomib (Velcade) and carfilzomib (Kyprolis) (73-75). As a peptide boronate, bortezomib showed great efficacy in multiple myelomas rather than in solid cancers (73). Bortezomib stabilizes I- $\kappa \mathrm{B}$, an important suppressor of $\mathrm{NF}^{-} \kappa \mathrm{B}$ signaling (74). Also, bortezomib causes accumulation of the tumor suppressors p27KIP1 and p53 (75). Furthermore, bortezomib can accumulate the pro-apoptotic protein BAX and induce endoplasmic reticulum stress and oxidative stress, which ultimately activate apoptosis of cancer cells (74). The major challenges for proteasome inhibitors are acquired resistance and fewer efficacies in solid tumors. Nevertheless, it may be worthwhile testing several combinations of proteasome inhibitors and also targeting the immunoproteasome, which causes inflammation in cancer and other diseases.

\subsection{MDM2 as an attractive drug target}

As shown previously, MDM2 is a negative regulator of p53 and therefore has oncogenic potential. Nutlins are a family of cis-imidazoline drugs that have been tested in clinical trials as inhibitors of MDM2. Some nutlins demonstrated promising tumor-suppressor effects on cancer cells, but this is dependent on their p53 mutational status (76). Other inhibitors of the MDM2-p53 interaction have also been 
tested. For instance, MI-219 and RITA (reactivation of p53 and induction of tumor cell apoptosis), can block the interaction, leading to reactivation of cell cycle arrest and apoptosis. Mechanistically, MI-219 binds to MDM2, whereas RITA binds to p53 but not MDM2, hence it might interfere with other interactions of p53 regardless of p53 ubiquitination status (77). Despite the great efficacy of these inhibitors, one drawback is their dependence on p53 wild-type state, since mutant p53 protein is no longer ubiquitinated by MDM2 or stabilized further.

\subsection{IAP antagonists}

In normal homeostasis, SMAC/DIABLO is a mitochondrial protein released to bind and inactivate IAPs, thereby activating apoptosis events (78). To generate IAPs antagonists, initial efforts have been made to mimic the four NH2-terminus residues of active SMAC that bind and inactivate the Baculovirus IAP Repeat (BIR) domains of IAPs (48). Importantly, antagonists of IAPs induce a conformational change and formation of IAP dimers, which lead to auto-ligase activity, auto-ubiquitination and finally degradation (79). For example, IAP antagonists (GDC-0152, LCL161, HGS1029 and TL32711) have entered clinical trials due to their efficient ubiquitination of IAPs, causing stimulation of TNFR1-mediated signaling and cancer cell death (80).

\subsection{Outputs from preclinical and clinical trials}

The UPS degrades numerous tumor suppressor proteins. Hence, it is now obvious that inhibition of proteasome activity reactivates apoptosis by sparing proteins like P53 (73-75). Inhibitors of the UPS may also induce apoptosis indirectly by inhibiting NF- $\kappa \mathrm{B}$ activation (81), hence preventing various anti-apoptotic proteins. Bortezomib, the proteasome inhibitor, is the only effector of the ubiquitin pathway amongst current anticancer therapeutics (82). VELCADE® is the commercial name of bortezomib and is used to treat hematological malignancies (multiple myeloma and mantle cell lymphomas). This molecule has been studied extensively during preclinical and clinical development to assess its cytotoxicity and its preference for tumor cells (83). It is suggested that bortezomib inhibits angiogenesis (84), is effective in various combination therapies, and can increase sensitivity to traditional cytotoxic therapies (85). Despite these efficacies, bortezomib's therapeutic window is still narrow, since dose-limiting toxicities are just above the treatment dose. Moreover, since mutations in the 85 chymotrypsin-like catalytic subunit of the proteasome are frequent, resistance to bortezomib is becoming evident (86).

Currently, 2nd generation molecules are undergoing clinical assessment to widen the therapeutic avenue. Inhibitors, or in some instances activators, of UPS enzymes should be developable as specific antitumor agents with toxicity profiles superior to that of proteasome inhibitors. For instance, MLN4924, an adenosine sulfamate analogue, inhibits the E1 activating enzyme responsible for NEDDylation, the covalent addition of a ubiquitin-like protein (NEDD8) to specific target proteins including SCFSkp2 (87). Because NEDDylation of Skp2 results in pro-growth activity inhibition of this process by MLN4924 is an effective therapeutic strategy. MLN4924 is currently in Phase II clinical trials for hematologic malignancies (82). Additionally, the E2 conjugating enzyme Cdc34 drives ubiquitination of p27, and inhibition of p27 ubiquitination and subsequent degradation is suggested to prevent tumor cell cycle progression. A small molecule inhibitor of Cdc34, named CC0651, is in preclinical development as a potential anticancer agent (88).

The first in-human, phase I clinical trial of p28 (NSC745104) was conducted to inhibit p53 ubiquitination in patients with p53(+) metastatic solid tumors (89). p28 (NSC745104) is a 28-amino-acid fragment of the cupredoxin azurin and acts as a 
non-HDM2-mediated peptide inhibitor of p53 ubiquitination. Safety, tolerability, pharmacokinetics and preliminary activity of p28 were tested. p28 was tolerated with no significant adverse effects. The anti-tumor activity indicates a highly favorable therapeutic index and demonstrates high efficiency of this novel class of non-HDM2-mediated peptide inhibitors (89). Nevertheless, selective inhibition of a given E3 ligase affects a limited number of cellular events because E3 ligases are more specific in terms of target proteins. Most E3 enzymes do not possess a classic catalytic active site; instead they mediate protein-protein interactions between the charged E2 enzyme and the protein substrate. Consequently, the most prominent ligase-based drug discovery strategy to date has been the development of antagonists of E3-substrate binding. For instance, the two E3 ligase antagonists currently in clinical trials for cancer, RO5045337 (nutlin-3) and JNJ-26854165, target MDM2, which ubiquitinates p53 in cancer cells for degradation (90). The fact that there are two MDM2-p53 binding inhibitors and seven IAP antagonists in Phase I/II clinical trials suggests that E3 ligases constitute a viable drug discovery area (82).

\section{Molecular mechanisms of tumor-suppressing roles of UPS components}

\subsection{E3 ligases as tumor suppressor proteins}

\section{The von Hippel-Lindau tumor suppressor}

The von Hippel-Lindau tumor suppressor (pVHL; encoded by VHL) is named after hereditary cancers characterized by highly vascularized tumors such as renal cell cancer, pancreatic tumors and tumors of the retinal and central nervous system $(91,92)$. pVHL is a part of the VCB-Cul2-VHL Ub ligase complex, which mediates the ubiquitination of hypoxia-inducible factor-1a (HIF-1a). Once HIF-1a is hydroxylated on proline residues, it undergoes ubiquitination by pVHL and subsequent degradation by the UPS (93). Loss-of-function mutations and deep deletions of $V H L$ have been observed in various cancers, including, renal, brain, pancreatic and cervical tumors (Fig. 7). Consequently, these mutations cause low VHL expression in different cancer tissues. Also, due to these mutations, HIF-1a is no longer ubiquitinated and stabilized and is not degraded by the proteasome system, leading to stimulation of rapid vascularization and tumor growth progression (93). In hypoxia, HIF-1a induces expression of vascular endothelial growth factor (VEGF), glucose transporter 1, matrix metalloproteases (MMPs), SNAIL, TWIST and PDGF, thereby promoting angiogenesis, migration and metastasis (94). As an upstream regulator, UBE2S (Ub-conjugating enzyme E2S, also known as E2-EPF ubiquitin carrier protein (UCP)), is involved in ubiquitination of the VHL protein, leading to its proteasomal degradation (95). Consequently, overexpression of UBE2S contributes to increased degradation of VHL and increased HIF-1a expression and VEGF transcription, leading to increased proliferation and metastasis (96). Upregulation of UBE2S and HIF-1a, with low VHL expression, is detected in several carcinomas, including liver, colorectal, breast, pancreatic and prostate cancers (9799).

\section{STUB1 E3 ligase}

STIP1 Homology and U-Box Containing Protein 1 (STUB1) is an E3 ubiquitin ligase downregulated in various carcinomas as it ubiquitinates cell cycle regulators, such as c-Myc and SRC-3 (100). Analysis of TCGA data showed that STUB1 is altered in several cancers with several genetic aberrations including mutations, amplifications and deep deletions (Fig. 8). Loss-of-function mutations and deep deletions of STUB1 are thought to cause downregulation of STUB1 expression levels in different cancers. Depletion of STUB1, a molecular chaperone for protein quality, augments NF- $\kappa B$ signaling, anti-apoptotic proteins (Bcl-2) and AKT. Hence, STUB1 downregulation 
associates with survival, invasiveness and metastasis of breast and colorectal cancers $(101,102)$. However, in pancreatic cancer, STUB1 modulates the stability of EGFR through proteasomal degradation of the receptor tyrosine kinase (RTK). Also, STUB1 regulates the phosphorylation of Tyr845 and Tyr1068 of EGFR, to modulate downstream PI3K/AKT and Src/FAK/paxillin signaling. Therefore, downregulation of STUB1 increases EGFR signaling and sensitization of pancreatic cancer cells to RTK inhibitors like erlotinib, leading to apoptosis and tumor suppression (103). Clinical targeting of STUB1 is indispensable in tumors harboring NF- $\kappa \mathrm{B}$ signaling. However, due to the discrepancy of STUB1 expression levels in different tumors, further investigations are required to reveal the context-dependent functions of STUB1 in cancers.

\section{FBXW7 E3 ligase}

Similarly, F-Box and WD Repeat Domain Containing 7 (FBXW7) is downregulated in several malignancies (e.g. colorectal, breast and gastric cancers, as well as cholangiocarcinoma) and is correlated with poor prognosis and survival, and enhanced invasiveness and metastasis (104-106). Several loss-of-function mutations and deep deletions of $F B X W 7$ are found in several types of cancers, including colorectal and cervical malignancies (Fig. 9). These loss-of-function mutations and deletions cause the downregulation of FBXW7 expression and loss of its tumorsuppressing functions in several malignancies. FBXW7 is an E3 ligase and a component of the SCF complex (SKP1, CUL-1, F-box protein), which regulates the stability of oncogenic regulators such as c-Myc, cyclin E and Notch (107). In cholangiocarcinoma, FBXW7 regulates the stability of the mTOR protein; hence FBXW7 downregulation increases mTOR levels and enhances the metastasis of cholangiocarcinoma cells to distant organs like the liver and lung. Moreover, downregulation of FBXW7 sensitizes these tumors to mTOR inhibitors like rapamycin, which impairs tumor progression and activates apoptosis (106).

The upstream regulators of FBXW7 have also been investigated. Xu et al. (108) reported that FBXW2 is a substrate of B-TrCP1 and an E3 ligase for SKP2. Whereas B-TrCP1 promotes the ubiquitination of FBXW2 for proteasomal degradation, FBXW2 does the same to SKP2. Therefore, FBXW2 exerts a tumor-suppressing function by blocking the oncogenic functions of $B-T r C P 1$ and SKP2. B-transducin repeat-containing protein $1(B-\mathrm{TrCP} 1)$ is one of the prototypical and bestcharacterized mammalian F-box proteins. 8 - $\mathrm{TrCP} 1$ has an oncogenic function by targeting many tumor suppressor proteins like P53 (109), IkB (110), programmed cell death protein 4 (PDCD4) (111) and DEPTOR (112). Hence, B-TrCP1 is overexpressed and associated with poor prognosis in different types of cancer, including colorectal cancer, pancreatic cancer, hepatoblastomas and breast cancer (113-115). Collectively, these findings reveal an important interplay among $\mathrm{F}$-box proteins that causes the versatile nature of the components of Ub system.

Downstream targets of FBXW7 include several oncoproteins such as Notch, c-Myc, cyclin $\mathrm{E}$ and c-Jun, all of which have oncoprotein activity and are overexpressed in several human malignancies (116). Several cancer-associated mutations in FBXW7 and its substrates have been frequently detected in various types of human cancer, including breast cancer, colon cancer and T-cell acute lymphoblastic leukaemia (TALL) (107). Knockout of FBXW7 in mice leads to chromosomal instability, tumorigenesis and gain of stemness properties. Emerging evidence reveals that FBXW7 regulates stem cell self-renewal, differentiation, survival and multipotency in several types of stem cells with different origins (117). Furthermore, FBXW7 governs cellular apoptosis (programmed cell death) by targeting MCL1, a prosurvival BCL2 family member, for ubiquitination-mediated degradation. Loss of FBXW7 and overexpression of MCL1 are observed in leukemia cells. FBXW7- 
deficient cells are particularly sensitive to the multi-kinase inhibitor sorafenib but are resistant to the BCL2 antagonist ABT-737 (118). These findings establish MCL1 as a therapeutically relevant bypass survival mechanism that enables FBXW7deficient cells to evade apoptosis. Hence, MCL1-targeted treatment is a promising strategy for leukemia patients with FBXW7-deficiency.

\section{ZNRF3 E3 ligase}

The E3 ubiquitin ligase, zinc and ring finger 3 (ZNRF3), is another member of the E3 ligase family with a tumor suppressing function in different types of human cancer (119). ZNRF3 is a transmembrane E3 ubiquitin ligase that functions as an important regulator of cancer development. Downregulation of ZNRF3 protein is often observed in cancerous tissues vs normal counterparts, with poor tissue differentiation. In gastric adenocarcinoma cells, overexpression of ZNRF3 induces apoptosis, suppresses proliferation and downregulates the Wnt/6-catenin/TCF signaling pathway (120). Similarly, in colorectal, gastric and papillary thyroid carcinomas, overexpression of ZNRF3 indicates a favorable prognosis by suppressing cancer cell growth and facilitating apoptosis $(120,121)$. Furthermore, ZNRF3's tumor-suppressing function is attributed to inactivation of both the Wnt and Hedgehog proliferative pathways $(122,123)$. In a recent study, Dong et al. (124) showed that R-spondin 2 (RSPO2) has an inhibitory effect on colorectal cancer cell migration, invasion and metastasis. RSPO2 interacts with Wnt receptor Frizzled 7 (Fzd7) to increase the degradation of Fzd7 via ZNRF3-mediated ubiquitination. These events lead to the suppression of the downstream PKC/ERK signaling in colorectal cancer (124). Therefore, reactivation of the ubiquitination potential of E3 ligases with tumor-suppressing functions (e.g. ZNRF3) would be of great importance clinically to destabilize aberrant oncogenic stability and signaling pathways involved in cancer cell proliferation and metastasis.

\section{FBXO31 and RNF144A E3 ligases}

The F-box protein FBXO31 is a component of the SCF E3 ubiquitin ligase complex and is downregulated in gastric cancer (125). Zou et al. (125) reported that FBXO31 suppresses cell cycle progression and EMT in gastric cancer cells by targeting Snail 1 for proteasomal degradation. The study showed that FBXO31 interacts with Snail1 and mediates the ubiquitin-dependent proteasomal degradation of Snaill in gastric cancer. Consistently, the study revealed a highly significant negative correlation between FBXO31 and Snail1 expression in human gastric cancer clinical specimens (125). In addition, ring finger protein 144A (RNF144A), a RING-betweenRING (RBR)-type E3 ubiquitin ligase, has recently been reported to target poly (ADP-ribose) polymerase 1 (PARP1). PARP1 proteins are critical DNA repair proteins, which are upregulated in breast cancers. Zhang et al. (126) showed that RNF144A interacts with PARP1 through its carboxy-terminal region containing the transmembrane domain, and targets PARP1 for ubiquitination and subsequent proteasomal degradation. Collectively, these studies suggest activators of tumor suppressor E3 ligases as a valid strategy in cancer therapy.

\section{Ubiquitination facilitator proteins}

Yes-associated protein 1 (YAP1) is a key transcription co-activator of the Hippo pathway, which is essential for cancer initiation, progression, or metastasis in clear cell renal cell carcinoma (ccRCC). Recently, Xiao et al. (127) identified YAP1 as an interactor protein with two members of the a-arrestin family (ARRDC1/3) in RCC cells through the WW domains of YAP1 and the PPXY motifs of ARRDC1/3. The study revealed that ARRDC1/3 mRNA levels are significantly downregulated in ccRCC specimens. Also, expression of ARRDC1/3 suppresses cancer cell growth, 
migration, invasion and EMT. Moreover, the study demonstrated that these effects are mediated, at least in part, through YAP1 degradation via ubiquitination. ARRDC1/3 negatively regulates YAP1 protein stability by facilitating E3 ubiquitin ligase Itch-mediated ubiquitination and degradation of YAP1. This study explains the relationship between ARRDC1/3 downregulation and aberrant Hippo-YAP1 pathway activation in ccRCC (127).

\subsection{E3 ligases with both tumor promoting and suppressing functions}

Cullin 3-RING ligases (CRL3s) are the largest E3 ligase family in eukaryotes and are multi-protein complexes. CRL3s exert pivotal roles in the regulation of various physiological and pathological processes, including cancer (128). CRL3s contain a BTB/POZ domain that triggers the interaction between Cullin 3 and target substrates to promote their ubiquitination and subsequent degradation (129). The biological implications of CRL3s are diverse insofar as they can function as either oncogenes or tumor suppressors or can mediate either of these effects in a contextdependent manner (129). For example, Keap1 likely serves as a tumor suppressor in different types of cancer, mainly due to its function to target its downstream oncogenic substrate, NRF2 (nuclear factor erythroid 2-related factor 2) (130). However, the role of the adaptor protein SPOP (speckle type BTB/POZ protein) in tumorigenesis appears to be tissue- and context-dependent. SPOP acts as a tumor suppressor via destabilizing downstream targets with oncogenic functions in several cancers, especially in prostate cancer (131). The downstream substrates of SPOP in prostate cancer include several oncoproteins such as c-MYC, EglN prolyl hydroxylases (132), Cdc20 (133), ERG oncoprotein (134) and SENP7 deSUMOylase (135). Moreover, Cullin 3/SPOP promotes the poly-ubiquitination and degradation of HDAC6; therefore, SPOP loss-of-function mutations might lead to metastasis in various human cancers (136). On the other hand, SPOP exerts an oncogenic function in kidney cancer by acting as a key regulatory hub (137) for the activation of the Bcatenin/TCF4 complex (138). Several studies have reported that somatic mutations of SPOP are detected in gastric, colorectal and prostate cancers. Mutations of SPOP involved the SPOP substrate-binding cleft, thereby impeding the Cul3-based ubiquitination of the substrate (139). Analysis of TCGA data showed that SPOP is altered in several malignancies via several genetic aberrations, including loss-offunction mutations and deep deletions (Fig. 10). Loss-of-function mutations of SPOP are thought to cause downregulation of SPOP expression levels in different cancers, especially prostate cancer. Moreover, it has recently been reported that prostate cancer-associated SPOP mutations confer resistance to BET inhibitors through stabilization of bromodomain 4 (BRD4) (140) and AKT-mTORC1 activation (141).

\subsection{Tumor-suppressor deubiquitinating enzymes}

DUBs remove Ub moieties from substrate proteins to prevent their degradation by the proteasome system. When the targeted substrate is acting as a tumor suppressor, DUBs function to stabilize these substrates and hence act as tumor suppressors. For instance, A20, CYLD and BAP1 are DUBs mutated in several types of cancer, especially immunological malignancies $(142,143)$. A20 interacts with proteins mediating the proinflammatory signaling pathways, such as tumor necrosis factor receptor (TNFR)-associated factor 2 (TRAF2), TRAF6 and NF-kB-essential modulator (NEMO). Consequently, A20 attenuates NF-kB signaling pathways by not only deubiquitination but also by Ub-editing through removal of $\mathrm{Ub}$ and generation of new Ub adducts. Therefore, A20 inactivation contributes to malignancy by promoting NF- $\mathrm{kB}$ signaling (142). A similar example is BAP1, which shows a similar activity by modulating the stability of epigenetic regulators. Aberrant expression and mutation of the BAP1-regulated polycomb protein ASXL1 are found in chronic myelomonocytic leukemia (143). Another exciting example is CYLD, which is 
mutated in multiple myelomas, lymphoblastic leukemias and melanomas. CYLD mutation leads to CYLD inactivation or downregulation, thereby increasing B-cell lymphoma $3\left(\mathrm{Bcl}^{-} 3\right)$ ubiquitination and nuclear localization and NF- $\mathrm{kB}$ activation (144). These effects ultimately lead to heightened invasion and proliferation.

\section{Reactivation of ubiquitination as a therapeutic approach in cancer}

\subsection{Targeting tumor-promoting deubiquitinases}

As shown previously, several DUBs are deregulated in cancer, leading to abnormal functions of their substrates. Therefore, inhibitors of USPs and other DUBs provide an excellent approach to restore the functions of tumor suppressors (e.g. PTEN and p53) by inducing their stability or modulating their activity in cancer cells. For instance, small molecule inhibitors of USP7 (e.g. HBX41\&108) reverse EMT and induce apoptosis in cancer cells (145). Importantly, these inhibitors reactivate ubiquitination-mediated nuclear inclusion of PTEN and restore the wild-type form of p53 (146). Some USP7 inhibitors are in preclinical development, but no compound has entered clinical trial. This interesting gain-of-function approach provides the basis for indispensable potential future studies for mutant p53-targeted therapies.

\subsection{Chemical reactivation of ubiquitination}

Several oncoproteins that are compromised in cancer, such as MYC, B-catenin and MCL1, are subject to ubiquitination and proteasomal degradation (147). Due to their robust activity, they are dubbed 'undruggable targets' in cancer. Hence, a promising therapeutic strategy is to reactivate the ubiquitination and subsequent degradation of these proteins to block tumorigenesis. These strategies include protein-targeting chimeric molecules (PROTACs) and hydrophobic tagging (HyT) (17). PROTACs are based on the concept of generating artificial molecules able to recruit a specific ubiquitin ligase and another molecule able to bind to the target protein itself (Fig. 11A). Therefore, PROTACs consist of a ligase-recruiting moiety linked through a short linker to a target protein-binding ligand. For example, PROTAC-1 acts to inhibit angiogenesis by harboring an angiogenesis inhibitor, ovalicin, a covalent binder for the methionine aminopeptidase-2 (MetAP-2), and an IkB phospho-peptide that is recognized by the E3 ligase, SCFB-TRCP. Therefore, MetAP-2 is recruited to SCFB-TRCP, ubiquitinated and degraded in a PROTAC-1-dependent manner (148).

On the other hand, hydrophobic tagging is based on the concept of misfolding of targeted proteins. A major driving force for correct protein folding is to minimize the number of hydrophobic moieties exposed to water. When protein misfolding occurs, cellular quality control machinery is engaged to induce protein degradation (149). This machinery can be deployed in cancer to remove unwanted oncoproteins by tagging them with a synthetic hydrophobic tag, thereby recruiting quality control machinery to initiate their proteasomal degradation (150) (Fig. 11B). For example, the hydrophobic group adamantine is linked to a target protein-binding moiety; adamantine thereby mimics or induces a misfolded state, resulting in ubiquitinproteasome mediated degradation (150). These emerging degrader technologies offer new vistas to target the 'undruggable' oncoproteins. Moreover, the activated degradation of oncoproteins by this strategy might also increase peptide presentation by the major histocompatibility complex (MHC) molecules, thereby synergizing with cancer immunotherapy. These technical advances would be of great clinical importance in combatting the drug resistance often seen in cancer.

\section{Conclusions and future perspectives}


The process of ubiquitination has a broad spectrum and diverse functions in both normal homeostasis and disease. Aberrant expression and mutations in components of the Ub network have been implicated in several types of cancer. Additionally, cancer cells may take advantage of a combinatorial deregulated expression of these components in order to support oncogenic signaling pathways. The function and ultimate effect of ubiquitination depends mainly on the nature of the effector substrate and type of ubiquitination-mediated effect. More specifically for cancer, the functions of ubiquitination include either tumor-promoting or tumor-suppressing effects. In efforts to tackle this deregulation, several strategies have been employed, ranging from inhibition to reactivation and enhancement of ubiquitination, depending on its nature as either friend or foe in cancer. Nevertheless, extra diseasecausing mutations within the Ub system components doubtlessly await discovery, depending on the technical advances in the detection of ubiquitination substrates and receptors.

Interestingly, dissection of the atypical Ub chains in the ubiquitinated proteins holds great promise. In particular, linear and branched chains of Ub mediate differential functions (Fig. 2). A great effort to decipher the signaling cascade and physiological roles of these chains is needed to unravel mechanistic insights in inflammation, cancer, autoimmunity and immune disorders. Future advances in targeting and inhibiting the protein-protein interactions could also permit the interference with binding of Ub to conjugation enzymes or Ub receptors. This could allow editing of ubiquitination chains and redirecting functional consequences. Moreover, by using phage display methods, researchers have generated Ub variants able to block actions of Ub ligases, DUBs and several Ub receptors (151). The same approach could be used to destabilize oncoproteins by dissociating them from DUBs or to stabilize tumor suppressor proteins by inhibiting degradation-promoting components of the Ub system. Coupled with the emerging degrader technologies (PROTACs \& HyT) described previously, these approaches could generate novel therapeutics for the treatment of human diseases, including cancer.

\section{Acknowledgements}

I thank Angela Kelsey, Catherine Winchester and Mark Nakasone at the Beatson Institute for their critical reading and editing of the manuscript. I thank Dr David Bryant at the Institute of Cancer Sciences for his encouragement and useful discussion. I apologize to all researchers whose crucial contributions in the field of ubiquitination were not referenced in this review, due to limitations in space. The author was supported by a Royal Society Newton International Fellowship.

\section{Conflict of interest}

The author declares no conflict of interest regarding financial and/or personal relationships with other people or organizations that could inappropriately influence (bias) this work.

\section{References}

1) Hershko A. Ubiquitin: roles in protein modification and breakdown. Cell. 1983;34(1):11-2.

2) Wilkinson KD. Protein ubiquitination: a regulatory post-translational modification. Anticancer Drug Des. 1987;2(2):211-29.

3) Wang D, Ma L, Wang B, Liu J, Wei W. E3 ubiquitin ligases in cancer and implications for therapies. Cancer Metastasis Rev. 2017;36(4):683-702. 
4) Schulman BA, Harper JW. Ubiquitin-like protein activation by E1 enzymes: the apex for downstream signalling pathways. Nat Rev Mol Cell Biol. 2009;10(5):31931.

5) D'arcy $\mathrm{P}$, Wang $\mathrm{X}$, Linder S. Deubiquitinase inhibition as a cancer therapeutic strategy. Pharmacol Ther. 2015;147:32-54.

6) Popovic D, Vucic D, Dikic I. Ubiquitination in disease pathogenesis and treatment. Nat Med. 2014;20(11):1242-53.

7) Ikeda F, Dikic I. Atypical ubiquitin chains: new molecular signals. 'Protein Modifications: Beyond the Usual Suspects' review series. EMBO Rep. 2008;9(6):536-42.

8) Bennett EJ, Harper JW. DNA damage: ubiquitin marks the spot. Nat Struct Mol Biol. 2008;15(1):20-2.

9) Pickart CM, Eddins MJ. Ubiquitin: structures, functions, mechanisms. Biochim Biophys Acta. 2004;1695(1-3):55-72.

10) Chen ZJ, Sun LJ. Nonproteolytic functions of ubiquitin in cell signaling. Mol Cell. 2009;33(3):275-86.

11) Bhattacharjee S, Nandi S. DNA damage response and cancer therapeutics through the lens of the Fanconi Anemia DNA repair pathway. Cell Commun Signal. 2017;15(1):41.

12) Martín-vicente M, Medrano LM, Resino S, García-sastre A, Martínez I. TRIM25 in the Regulation of the Antiviral Innate Immunity. Front Immunol. 2017;8:1187.

13) Kattah MG, Malynn BA, Ma A. Ubiquitin-Modifying Enzymes and Regulation of the Inflammasome. J Mol Biol. 2017;429(22):3471-3485.

14) $\mathrm{Lu} \mathrm{Z}$, Hunter T. Ubiquitylation and proteasomal degradation of the p21(Cip1), p27(Kip1) and p57(Kip2) CDK inhibitors. Cell Cycle. 2010;9(12):2342-52.

15) Love IM, Shi D, Grossman SR. p53 Ubiquitination and proteasomal degradation. Methods Mol Biol. 2013;962:63-73.

16) Paul I, Batth TS, Iglesias-gato D, et al. The ubiquitin ligase Cullin5SOCS2 regulates NDR1/STK38 stability and NF-kB transactivation. Sci Rep. $2017 ; 7: 42800$.

17) Huang X, Dixit VM. Drugging the undruggables: exploring the ubiquitin system for drug development. Cell Res. 2016;26(4):484-98.

18) Hoeller D, Dikic I. Targeting the ubiquitin system in cancer therapy. Nature. 2009;458(7237):438-44.

19) Lipkowitz S, Weissman AM. RINGs of good and evil: RING finger ubiquitin ligases at the crossroads of tumour suppression and oncogenesis. Nat Rev Cancer. 2011;11(9):629-43. 
20) Kirkin V, Dikic I. Ubiquitin networks in cancer. Curr Opin Genet Dev. 2011;21(1):21-8.

21) Momand J, Zambetti GP, Olson DC, George D, Levine AJ. The mdm-2 oncogene product forms a complex with the p53 protein and inhibits p53-mediated transactivation. Cell. 1992;69(7):1237-45.

22) Kussie PH, Gorina S, Marechal V, et al. Structure of the MDM2 oncoprotein bound to the p53 tumor suppressor transactivation domain. Science. 1996;274(5289):948-53.

23) Haupt Y, Maya R, Kazaz A, Oren M. Mdm2 promotes the rapid degradation of p53. Nature. 1997;387(6630):296-9.

24) Quesnel B, Preudhomme C, Oscier D, et al. Over-expression of the MDM2 gene is found in some cases of haematological malignancies. $\mathrm{Br} J$ Haematol. $1994 ; 88(2): 415-8$.

25) Mccann AH, Kirley A, Carney DN, et al. Amplification of the MDM2 gene in human breast cancer and its association with MDM2 and p53 protein status. Br J Cancer. 1995;71(5):981-5.

26) Shen J, Li P, Shao X, et al. The E3 Ligase RING1 Targets p53 for Degradation and Promotes Cancer Cell Proliferation and Survival. Cancer Res. 2018;78(2):359-371.

27) Xiong Y, Hu B, Wei L, Jiang D, Zhu M. Upregulated expression of polycomb protein Ring1 contributes to poor prognosis and accelerated proliferation in human hepatocellular carcinoma. Tumour Biol. 2015;36(12):9579-88.

28) Carrano AC, Eytan E, Hershko A, Pagano M. SKP2 is required for ubiquitinmediated degradation of the CDK inhibitor p27. Nat Cell Biol. 1999;1(4):193-9.

29) Sutterlüty H, Chatelain E, Marti A, et al. p45SKP2 promotes p27Kip1 degradation and induces S phase in quiescent cells. Nat Cell Biol. 1999;1(4):20714.

30) $\mathrm{Yu}$ ZK, Gervais JL, Zhang H. Human CUL-1 associates with the SKP1/SKP2 complex and regulates p21(CIP1/WAF1) and cyclin D proteins. Proc Natl Acad Sci USA. 1998;95(19):11324-9.

31) Kamura T, Hara T, Kotoshiba S, et al. Degradation of p57Kip2 mediated by SCFSkp2-dependent ubiquitylation. Proc Natl Acad Sci USA. 2003;100(18):10231-6.

32) Tedesco D, Lukas J, Reed SI. The pRb-related protein p130 is regulated by phosphorylation-dependent proteolysis via the protein-ubiquitin ligase SCF(Skp2). Genes Dev. 2002;16(22):2946-57.

33) Huang H, Regan KM, Wang F, et al. Skp2 inhibits FOXO1 in tumor suppression through ubiquitin-mediated degradation. Proc Natl Acad Sci USA. 2005;102(5):1649-54. 
34) Chan $\mathrm{CH}$, Li CF, Yang WL, et al. The Skp2-SCF E3 ligase regulates Akt ubiquitination, glycolysis, herceptin sensitivity, and tumorigenesis. Cell. 2012;149(5):1098-111.

35) Souers AJ, Leverson JD, Boghaert ER, et al. ABT-199, a potent and selective BCL-2 inhibitor, achieves antitumor activity while sparing platelets. Nat Med. 2013;19(2):202-8.

36) Wu L, Grigoryan AV, Li Y, Hao B, Pagano M, Cardozo TJ. Specific small molecule inhibitors of Skp2-mediated p27 degradation. Chem Biol. 2012;19(12):1515-24.

37) Chan CH, Morrow JK, Li CF, et al. Pharmacological inactivation of Skp2 SCF ubiquitin ligase restricts cancer stem cell traits and cancer progression. Cell. 2013;154(3):556-68.

38) Shi C, Pan BQ, Shi F, et al. Sequestosome 1 protects esophageal squamous carcinoma cells from apoptosis via stabilizing SKP2 under serum starvation condition. Oncogene. 2018; doi: 10.1038/s41388-018-0217-0.

39) Su L, Han D, Wu J, Huo X. Skp2 regulates non-small cell lung cancer cell growth by Meg3 and miR-3163. Tumour Biol. 2016;37(3):3925-31.

40) Evans L, Chen L, Milazzo G, et al. SKP2 is a direct transcriptional target of MYCN and a potential therapeutic target in neuroblastoma. Cancer Lett. 2015;363(1):37-45.

41) Wang JY, Liu GZ, Wilmott JS, et al. Skp2-Mediated Stabilization of MTH1 Promotes Survival of Melanoma Cells upon Oxidative Stress. Cancer Res. 2017;77(22):6226-6239.

42) Dai X, Guo G, Zou P, et al. (S)-crizotinib induces apoptosis in human non-small cell lung cancer cells by activating ROS independent of MTH1. J Exp Clin Cancer Res. 2017;36(1):120.

43) Lu W, Liu S, Li B, et al. SKP2 loss destabilizes EZH2 by promoting TRAF6mediated ubiquitination to suppress prostate cancer. Oncogene. 2017;36(10):1364-1373.

44) Jiang N, Ke B, Hjort-jensen K, et al. YAP1 regulates prostate cancer stem celllike characteristics to promote castration resistant growth. Oncotarget. 2017;8(70):115054-115067.

45) Deveraux QL, Reed JC. IAP family proteins--suppressors of apoptosis. Genes Dev. 1999;13(3):239-52.

46) Vaux DL, Silke J. IAPs, RINGs and ubiquitylation. Nat Rev Mol Cell Biol. 2005;6(4):287-97.

47) Silke J, Vucic D. IAP family of cell death and signaling regulators. Meth Enzymol. 2014;545:35-65.

48) Varfolomeev E, Blankenship JW, Wayson SM, et al. IAP antagonists induce autoubiquitination of c-IAPs, NF-kappaB activation, and TNFalpha-dependent apoptosis. Cell. 2007;131(4):669-81. 
49) Zender L, Spector MS, Xue W, et al. Identification and validation of oncogenes in liver cancer using an integrative oncogenomic approach. Cell. 2006;125(7):125367.

50) Xu L, Lubkov V, Taylor LJ, Bar-sagi D. Feedback regulation of Ras signaling by Rabex-5-mediated ubiquitination. Curr Biol. 2010;20(15):1372-7.

51) Hobbs GA, Gunawardena HP, Baker R, Campbell SL. Site-specific monoubiquitination activates Ras by impeding GTPase-activating protein function. Small GTPases. 2013;4(3):186-92.

52) Bournet B, Muscari F, Buscail C, et al. KRAS G12D Mutation Subtype Is A Prognostic Factor for Advanced Pancreatic Adenocarcinoma. Clin Transl Gastroenterol. 2016;7:e157.

53) Farhan M, Wang H, Gaur U, Little PJ, Xu J, Zheng W. FOXO Signaling Pathways as Therapeutic Targets in Cancer. Int J Biol Sci. 2017;13(7):815-827.

54) Mansour MA, Asano E, Hyodo T, et al. Special AT-rich sequence-binding protein 2 suppresses invadopodia formation in HCT116 cells via palladin inhibition. Exp Cell Res. 2015;332(1):78-88.

55) Mansour MA, Hyodo T, Ito S, et al. SATB2 suppresses the progression of colorectal cancer cells via inactivation of MEK5/ERK5 signaling. FEBS J. 2015;282(8):1394-405.

56) Akter KA, Mansour MA, Hyodo T, Ito S, Hamaguchi M, Senga T. FAM98A is a novel substrate of PRMT1 required for tumor cell migration, invasion, and colony formation. Tumour Biol. 2016;37(4):4531-9.

57) Mansour MA, Hyodo T, Akter KA, et al. SATB1 and SATB2 play opposing roles in c-Myc expression and progression of colorectal cancer. Oncotarget. 2016;7(4):4993-5006.

58) Akter KA, Mansour MA, Hyodo T, Senga T. FAM98A associates with DDX1C14orf166-FAM98B in a novel complex involved in colorectal cancer progression. Int J Biochem Cell Biol. 2016;84:1-13.

59) Kurita K, Maeda M, Mansour MA, et al. TRIP13 is expressed in colorectal cancer and promotes cancer cell invasion. Oncol Lett. 2016;12(6):5240-5246.

60) Mansour MA, Senga T. HOXD8 exerts a tumor-suppressing role in colorectal cancer as an apoptotic inducer. Int J Biochem Cell Biol. 2017;88:1-13.

61) Gallo LH, Ko J, Donoghue DJ. The importance of regulatory ubiquitination in cancer and metastasis. Cell Cycle. 2017;16(7):634-648.

62) Vaughan L, Tan CT, Chapman A, et al. HUWE1 ubiquitylates and degrades the RAC activator TIAM1 promoting cell-cell adhesion disassembly, migration, and invasion. Cell Rep. 2015;10(1):88-102.

63) Tsai YC, Mendoza A, Mariano JM, et al. The ubiquitin ligase gp78 promotes sarcoma metastasis by targeting KAI1 for degradation. Nat Med. 2007;13(12):1504-9. 
64) Jing Y, Nguyen MM, Wang D, et al. DHX15 promotes prostate cancer progression by stimulating Siah2-mediated ubiquitination of androgen receptor. Oncogene. 2018;37(5):638-650.

65) Zhao K, Yang Y, Zhang G, et al. Regulation of the Mdm2-p53 pathway by the ubiquitin E3 ligase MARCH7. EMBO Rep. 2018;

66) Ma X, Zhao J, Yang F, Liu H, Qi W. Ubiquitin conjugating enzyme E2 L3 promoted tumor growth of NSCLC through accelerating p27kip1 ubiquitination and degradation. Oncotarget. 2017;8(48):84193-84203.

67) Williams SA, Maecker HL, French DM, et al. USP1 deubiquitinates ID proteins to preserve a mesenchymal stem cell program in osteosarcoma. Cell. 2011;146(6):918-30.

68) Pereg Y, Liu BY, O'rourke KM, et al. Ubiquitin hydrolase Dub3 promotes oncogenic transformation by stabilizing Cdc25A. Nat Cell Biol. 2010;12(4):400-6.

69) Nijman SM, Luna-vargas MP, Velds A, et al. A genomic and functional inventory of deubiquitinating enzymes. Cell. 2005;123(5):773-86.

70) Zhang L, Zhou F, Drabsch Y, et al. USP4 is regulated by AKT phosphorylation and directly deubiquitylates TGF- $B$ type I receptor. Nat Cell Biol. 2012;14(7):71726.

71) Matsuura I, Lai CY, Chiang KN. Functional interaction between Smad3 and S100A4 (metastatin-1) for TGF-beta-mediated cancer cell invasiveness. Biochem J. 2010;426(3):327-35.

72) Song MS, Salmena L, Carracedo A, et al. The deubiquitinylation and localization of PTEN are regulated by a HAUSP-PML network. Nature. 2008;455(7214):8137.

73) Hideshima T, Richardson P, Chauhan D, et al. The proteasome inhibitor PS-341 inhibits growth, induces apoptosis, and overcomes drug resistance in human multiple myeloma cells. Cancer Res. 2001;61(7):3071-6.

74) Chauhan D, Hideshima T, Mitsiades C, Richardson P, Anderson KC. Proteasome inhibitor therapy in multiple myeloma. Mol Cancer Ther. 2005;4(4):686-92.

75) Hideshima T, Mitsiades C, Akiyama M, et al. Molecular mechanisms mediating antimyeloma activity of proteasome inhibitor PS-341. Blood. 2003;101(4):1530-4.

76) Sosin AM, Burger AM, Siddiqi A, Abrams J, Mohammad RM, Al-katib AM. HDM2 antagonist MI-219 (spiro-oxindole), but not Nutlin-3 (cis-imidazoline), regulates p53 through enhanced HDM2 autoubiquitination and degradation in human malignant B-cell lymphomas. J Hematol Oncol. 2012;5:57.

77) Issaeva N, Bozko P, Enge M, et al. Small molecule RITA binds to p53, blocks p53HDM-2 interaction and activates p53 function in tumors. Nat Med. 2004;10(12):1321-8. 
78) Verhagen AM, Ekert PG, Pakusch M, et al. Identification of DIABLO, a mammalian protein that promotes apoptosis by binding to and antagonizing IAP proteins. Cell. 2000;102(1):43-53.

79) Dueber EC, Schoeffler AJ, Lingel A, et al. Antagonists induce a conformational change in cIAP1 that promotes autoubiquitination. Science. 2011;334(6054):37680 .

80) Fulda S, Vucic D. Targeting IAP proteins for therapeutic intervention in cancer. Nat Rev Drug Discov. 2012;11(2):109-24.

81) Hideshima T, Chauhan D, Richardson $\mathrm{P}$, et al. NF-kappa B as a therapeutic target in multiple myeloma. J Biol Chem. 2002;277(19):16639-47.

82) Mattern $\mathrm{MR}, \mathrm{Wu}$ J, Nicholson B. Ubiquitin-based anticancer therapy: carpet bombing with proteasome inhibitors vs surgical strikes with E1, E2, E3, or DUB inhibitors. Biochim Biophys Acta. 2012;1823(11):2014-21.

83) Adams J. The development of proteasome inhibitors as anticancer drugs. Cancer Cell. 2004;5(5):417-21.

84) Williams S, Pettaway C, Song R, Papandreou C, Logothetis C, Mcconkey DJ. Differential effects of the proteasome inhibitor bortezomib on apoptosis and angiogenesis in human prostate tumor xenografts. Mol Cancer Ther. 2003;2(9):835-43.

85) Mitsiades N, Mitsiades CS, Richardson PG, et al. The proteasome inhibitor PS341 potentiates sensitivity of multiple myeloma cells to conventional chemotherapeutic agents: therapeutic applications. Blood. 2003;101(6):2377-80.

86) Suzuki E, Demo S, Deu E, et al. Molecular mechanisms of bortezomib resistant adenocarcinoma cells. PLoS ONE. 2011;6(12):e27996.

87) Chen Q, Xie W, Kuhn DJ, et al. Targeting the p27 E3 ligase SCF(Skp2) results in p27- and Skp2-mediated cell-cycle arrest and activation of autophagy. Blood. 2008;111(9):4690-9.

88) Ceccarelli DF, Tang X, Pelletier B, et al. An allosteric inhibitor of the human Cdc34 ubiquitin-conjugating enzyme. Cell. 2011;145(7):1075-87.

89) Warso MA, Richards JM, Mehta D, et al. A first-in-class, first-in-human, phase I trial of p28, a non-HDM2-mediated peptide inhibitor of p53 ubiquitination in patients with advanced solid tumours. Br J Cancer. 2013;108(5):1061-70.

90) Yuan Y, Liao YM, Hsueh CT, Mirshahidi HR. Novel targeted therapeutics: inhibitors of MDM2, ALK and PARP. J Hematol Oncol. 2011;4:16.

91) Gnarra JR, Tory K, Weng Y, et al. Mutations of the VHL tumour suppressor gene in renal carcinoma. Nat Genet. 1994;7(1):85-90.

92) Kanno H, Kondo K, Ito S, et al. Somatic mutations of the von Hippel-Lindau tumor suppressor gene in sporadic central nervous system hemangioblastomas. Cancer Res. 1994;54(18):4845-7. 
93) Maxwell PH, Wiesener MS, Chang GW, et al. The tumour suppressor protein VHL targets hypoxia-inducible factors for oxygen-dependent proteolysis. Nature. 1999;399(6733):271-5.

94) Singh D, Arora R, Kaur P, Singh B, Mannan R, Arora S. Overexpression of hypoxia-inducible factor and metabolic pathways: possible targets of cancer. Cell Biosci. 2017;7:62.

95) Jung CR, Hwang KS, Yoo J, et al. E2-EPF UCP targets pVHL for degradation and associates with tumor growth and metastasis. Nat Med. 2006;12(7):809-16.

96) Rankin EB, Giaccia AJ. Hypoxic control of metastasis. Science. 2016;352(6282):175-80.

97) Witkiewicz AK, Mcmillan EA, Balaji U, et al. Whole-exome sequencing of pancreatic cancer defines genetic diversity and therapeutic targets. Nat Commun. 2015;6:6744.

98) Beltran H, Prandi D, Mosquera JM, et al. Divergent clonal evolution of castration-resistant neuroendocrine prostate cancer. Nat Med. 2016;22(3):298305.

99) Ayesha AK, Hyodo T, Asano E, et al. UBE2S is associated with malignant characteristics of breast cancer cells. Tumour Biol. 2016;37(1):763-72.

100) Kajiro M, Hirota R, Nakajima $Y$, et al. The ubiquitin ligase CHIP acts as an upstream regulator of oncogenic pathways. Nat Cell Biol. 2009;11(3):312-9.

101) Wang $\mathrm{Y}$, Ren F, Wang $\mathrm{Y}$, et al. CHIP/Stub1 functions as a tumor suppressor and represses NF-kB-mediated signaling in colorectal cancer. Carcinogenesis. 2014;35(5):983-91.

102) Hiyoshi H, Goto N, Tsuchiya M, et al. 2-(4-Hydroxy-3-methoxyphenyl)benzothiazole suppresses tumor progression and metastatic potential of breast cancer cells by inducing ubiquitin ligase CHIP. Sci Rep. 2014;4:7095.

103) Wang T, Yang J, Xu J, et al. CHIP is a novel tumor suppressor in pancreatic cancer through targeting EGFR. Oncotarget. 2014;5(7):1969-86.

104) Ibusuki M, Yamamoto Y, Shinriki S, Ando Y, Iwase H. Reduced expression of ubiquitin ligase FBXW7 mRNA is associated with poor prognosis in breast cancer patients. Cancer Sci. 2011;102(2):439-45.

105) Iwatsuki M, Mimori K, Ishii H, et al. Loss of FBXW7, a cell cycle regulating gene, in colorectal cancer: clinical significance. Int J Cancer. 2010;126(8):1828-37.

106) Yang H, Lu X, Liu Z, et al. FBXW7 suppresses epithelial-mesenchymal transition, stemness and metastatic potential of cholangiocarcinoma cells. Oncotarget. 2015;6(8):6310-25.

107) Welcker M, Clurman BE. FBW7 ubiquitin ligase: a tumour suppressor at the crossroads of cell division, growth and differentiation. Nat Rev Cancer. 2008;8(2):83-93. 
108) Xu J, Zhou W, Yang F, et al. The B-TrCP-FBXW2-SKP2 axis regulates lung cancer cell growth with FBXW2 acting as a tumour suppressor. Nat Commun. 2017;8:14002.

109) Xia Y, Padre RC, De mendoza TH, Bottero V, Tergaonkar VB, Verma IM. Phosphorylation of p53 by IkappaB kinase 2 promotes its degradation by betaTrCP. Proc Natl Acad Sci USA. 2009;106(8):2629-34.

110) Winston JT, Strack P, Beer-romero P, Chu CY, Elledge SJ, Harper JW. The SCFbeta-TRCP-ubiquitin ligase complex associates specifically with phosphorylated destruction motifs in IkappaBalpha and beta-catenin and stimulates IkappaBalpha ubiquitination in vitro. Genes Dev. 1999;13(3):270-83.

111) Dorrello NV, Peschiaroli A, Guardavaccaro D, Colburn NH, Sherman NE, Pagano M. S6K1- and betaTRCP-mediated degradation of PDCD4 promotes protein translation and cell growth. Science. 2006;314(5798):467-71.

112) Gao D, Inuzuka $\mathrm{H}$, Tan $\mathrm{MK}$, et al. mTOR drives its own activation via $\mathrm{SCF}(B \mathrm{TrCP})$-dependent degradation of the mTOR inhibitor DEPTOR. Mol Cell. 2011;44(2):290-303.

113) Ougolkov A, Zhang B, Yamashita K, et al. Associations among beta-TrCP, an E3 ubiquitin ligase receptor, beta-catenin, and $\mathrm{NF}-k a p p a B$ in colorectal cancer. J Natl Cancer Inst. 2004;96(15):1161-70.

114) Müerköster S, Arlt A, Sipos B, et al. Increased expression of the E3-ubiquitin ligase receptor subunit betaTRCP1 relates to constitutive nuclear factor-kappaB activation and chemoresistance in pancreatic carcinoma cells. Cancer Res. 2005;65(4):1316-24.

115) Koch A, Waha A, Hartmann W, et al. Elevated expression of Wnt antagonists is a common event in hepatoblastomas. Clin Cancer Res. 2005;11(12):4295-304.

116) Cao J, Ge MH, Ling ZQ. Fbxw7 Tumor Suppressor: A Vital Regulator Contributes to Human Tumorigenesis. Medicine (Baltimore). 2016;95(7):e2496.

117) Wang Z, Inuzuka H, Fukushima H, et al. Emerging roles of the FBW7 tumour suppressor in stem cell differentiation. EMBO Rep. 2011;13(1):36-43.

118) Inuzuka $\mathrm{H}$, Shaik $\mathrm{S}$, Onoyama I, et al. SCF(FBW7) regulates cellular apoptosis by targeting MCL1 for ubiquitylation and destruction. Nature. 2011;471(7336):104-9.

119) Qiu W, Yang Z, Fan Y, Zheng Q. ZNRF3 is downregulated in papillary thyroid carcinoma and suppresses the proliferation and invasion of papillary thyroid cancer cells. Tumour Biol. 2016;37(9):12665-12672.

120) Zhou Y, Lan J, Wang W, et al. ZNRF3 acts as a tumour suppressor by the Wnt signalling pathway in human gastric adenocarcinoma. $J$ Mol Histol. 2013;44(5):555-63.

121) Yu N, Zhu H, Tao Y, et al. Association between prognostic survival of human colorectal carcinoma and ZNRF3 expression. Onco Targets Ther. 2016;9:66796687. 
122) Qin H, Cai A, Xi H, Yuan J, Chen L. ZnRF3 Induces Apoptosis of Gastric Cancer Cells by Antagonizing Wnt and Hedgehog Signaling. Cell Biochem Biophys. 2015;73(2):361-367.

123) Wang Z, Wang Y, Ren H, Jin Y, Guo Y. ZNRF3 Inhibits the Invasion and Tumorigenesis in Nasopharyngeal Carcinoma Cells by Inactivating the Wnt/6Catenin Pathway. Oncol Res. 2017;25(4):571-577.

124) Dong X, Liao W, Zhang L, et al. RSPO2 suppresses colorectal cancer metastasis by counteracting the Wnt5a/Fzd7-driven noncanonical Wnt pathway. Cancer Lett. 2017;402:153-165.

125) Zou S, Ma C, Yang F, Xu X, Jia J, Liu Z. FBXO31 Suppresses Gastric Cancer EMT by Targeting Snail1 for Proteasomal Degradation. Mol Cancer Res. 2018;16(2):286-295.

126) Zhang Y, Liao XH, Xie HY, Shao ZM, Li DQ. RBR-type E3 ubiquitin ligase RNF144A targets PARP1 for ubiquitin-dependent degradation and regulates PARP inhibitor sensitivity in breast cancer cells. Oncotarget. 2017;8(55):9450594518.

127) Xiao J, Shi Q, Li W, et al. ARRDC1 and ARRDC3 act as tumor suppressors in renal cell carcinoma by facilitating YAP1 degradation. Am J Cancer Res. 2018;8(1):132-143.

128) Cheng J, Guo J, Wang Z, et al. Functional analysis of Cullin 3 E3 ligases in tumorigenesis. Biochim Biophys Acta. 2018;1869(1):11-28.

129) Bulatov E, Ciulli A. Targeting Cullin-RING E3 ubiquitin ligases for drug discovery: structure, assembly and small-molecule modulation. Biochem J. 2015;467(3):365-86.

130) Best SA, De souza DP, Kersbergen A, et al. Synergy between the KEAP1/NRF2 and PI3K Pathways Drives Non-Small-Cell Lung Cancer with an Altered Immune Microenvironment. Cell Metab. 2018;27(4):935-943.e4.

131) Shoag J, Liu D, Blattner M, et al. SPOP mutation drives prostate neoplasia without stabilizing oncogenic transcription factor ERG. $J$ Clin Invest. 2018;128(1):381-386.

132) Geng C, Kaochar S, Li M, et al. SPOP regulates prostate epithelial cell proliferation and promotes ubiquitination and turnover of c-MYC oncoprotein. Oncogene. 2017;36(33):4767-4777.

133) Zhang L, Peng S, Dai X, et al. Tumor suppressor SPOP ubiquitinates and degrades EglN2 to compromise growth of prostate cancer cells. Cancer Lett. 2017;390:11-20.

134) Gan W, Dai X, Lunardi A, et al. SPOP Promotes Ubiquitination and Degradation of the ERG Oncoprotein to Suppress Prostate Cancer Progression. Mol Cell. 2015;59(6):917-30. 
135) Zhu H, Ren S, Bitler BG, et al. SPOP E3 Ubiquitin Ligase Adaptor Promotes Cellular Senescence by Degrading the SENP7 deSUMOylase. Cell Rep. 2015;13(6):1183-1193.

136) Tan Y, Ci Y, Dai X, et al. Cullin 3SPOP ubiquitin E3 ligase promotes the poly-ubiquitination and degradation of HDAC6. Oncotarget. 2017;8(29):4789047901.

137) Li G, Ci W, Karmakar S, et al. SPOP promotes tumorigenesis by acting as a key regulatory hub in kidney cancer. Cancer Cell. 2014;25(4):455-68.

138) Zhao W, Zhou J, Deng Z, Gao Y, Cheng Y. SPOP promotes tumor progression via activation of 8 -catenin/TCF4 complex in clear cell renal cell carcinoma. Int $\mathrm{J}$ Oncol. 2016;49(3):1001-8.

139) Barbieri CE, Baca SC, Lawrence MS, et al. Exome sequencing identifies recurrent SPOP, FOXA1 and MED12 mutations in prostate cancer. Nat Genet. 2012;44(6):685-9.

140) Dai X, Gan W, Li X, et al. Prostate cancer-associated SPOP mutations confer resistance to BET inhibitors through stabilization of BRD4. Nat Med. 2017;23(9):1063-1071.

141) Zhang P, Wang D, Zhao Y, et al. Intrinsic BET inhibitor resistance in SPOPmutated prostate cancer is mediated by BET protein stabilization and AKTmTORC1 activation. Nat Med. 2017;23(9):1055-1062.

142) Hymowitz SG, Wertz IE. A20: from ubiquitin editing to tumour suppression. Nat Rev Cancer. 2010;10(5):332-41.

143) Dey A, Seshasayee D, Noubade R, et al. Loss of the tumor suppressor BAP1 causes myeloid transformation. Science. 2012;337(6101):1541-6.

144) Bonnet M, Courtois G. [CYLD deubiquitinase as a recurrent target in oncogenic processes]. Med Sci (Paris). 2011;27(6-7):626-31.

145) Colland F, Formstecher E, Jacq X, et al. Small-molecule inhibitor of USP7/HAUSP ubiquitin protease stabilizes and activates p53 in cells. Mol Cancer Ther. 2009;8(8):2286-95.

146) Rivlin N, Katz S, Doody M, et al. Rescue of embryonic stem cells from cellular transformation by proteomic stabilization of mutant p53 and conversion into WT conformation. Proc Natl Acad Sci USA. 2014;111(19):7006-11.

147) Dreas A, Mikulski M, Milik M, Fabritius CH, Brzózka K, Rzymski T. Mitogen-activated Protein Kinase (MAPK) Interacting Kinases 1 and 2 (MNK1 and MNK2) as Targets for Cancer Therapy: Recent Progress in the Development of MNK Inhibitors. Curr Med Chem. 2017;24(28):3025-3053.

148) Sakamoto KM, Kim KB, Kumagai A, Mercurio F, Crews CM, Deshaies RJ. Protacs: chimeric molecules that target proteins to the Skp1-Cullin-F box complex for ubiquitination and degradation. Proc Natl Acad Sci USA. 2001;98(15):8554-9. 
149) Mcclellan AJ, Tam S, Kaganovich D, Frydman J. Protein quality control: chaperones culling corrupt conformations. Nat Cell Biol. 2005;7(8):736-41.

150) Neklesa TK, Tae HS, Schneekloth AR, et al. Small-molecule hydrophobic tagging-induced degradation of HaloTag fusion proteins. Nat Chem Biol. 2011;7(8):538-43.

151) Iconomou M, Saunders DN. Systematic approaches to identify E3 ligase substrates. Biochem J. 2016;473(22):4083-4101.

\section{Figure legends}

Figure 1: Schematic representation of the ubiquitination reactions of proteins with components of the $\mathrm{Ub}$ system indicated. Ubiquitin is activated through a thioester bond with the activating enzyme E1 in an ATP-dependent mechanism. Ub is then transferred by enzymatic reactions including the activating (E1), conjugating (E2) and ligating (E3) enzymes to the target protein. Mono-Ub proteins undergo multiple ubiquitination steps, which target the protein for proteasomal degradation or modulate its function.

Figure 2: Schematic representation of the multiple ubiquitination steps of mono-Ub proteins and their functions. Mono-Ub and multi-mono-Ub proteins are implicated in endocytosis, DNA repair and protein localization and trafficking. Depending on the type of poly-Ub chain, the protein has different fates inside the cell.

Figure 3: Flow diagram of the current review contents.

Figure 4: Schematic representation of the MDM2-p53 interaction. P53 is ubiquitinated by MDM2 and undergoes degradation via the proteasome. Nonubiquitinated p53 forms a tetramer able to bind to DNA to mediate its tumor suppressor effect.

Figure 5: Analysis of data in The Cancer Genome Atlas (TCGA) cBioportal shows genetic alterations of $M D M 2$ in several types of cancer. Mutation, fusion, amplification, deep deletion and multiple alterations are represented in the graph with different colors.

Figure 6: Analysis of data in The Cancer Genome Atlas (TCGA) cBioportal shows genetic alterations of SKP2 in several types of cancer. Mutation, amplification, deep deletion and multiple alterations are represented in the graph with different colors.

Figure 7: Analysis of data in The Cancer Genome Atlas (TCGA) cBioportal shows genetic alterations of $V H L$ in several types of cancer. Mutation, amplification and deep deletion are represented in the graph with different colors.

Figure 8: Analysis of data in The Cancer Genome Atlas (TCGA) cBioportal shows genetic alterations of STUB1 in several types of cancer. Mutation, amplification and deep deletion are represented in the graph with different colors.

Figure 9: Analysis of data in The Cancer Genome Atlas (TCGA) cBioportal shows genetic alterations of $F B X W 7$ in several types of cancer. Mutation, fusion, amplification, deep deletion and multiple alterations are represented in the graph with different colors. 
Figure 10: Analysis of data in The Cancer Genome Atlas (TCGA) cBioportal shows genetic alterations of SPOP in several types of cancer. Mutation, amplification and deep deletion are represented in the graph with different colors.

Figure 11: Schematic representation of the advanced techniques to reactivate ubiquitination of target proteins. (A) protein-targeting chimeric molecules (PROTACs) are bifunctional molecules comprised of a targeting ligand tethered to an E3 ligase-recruiting moiety through a short linker. (B) Hydrophobic tagging (HyT) molecules are bifunctional molecules that are comprised of a substrate-recruiting ligand connected with a hydrophobic moiety (e.g. adamantine) through a short linker. Hydrophobic tagging of proteins mimics the partially unfolded state, triggering the recruitment of the chaperone machinery to drive protein degradation directly via the proteasome or indirectly through initiating ubiquitination of the target protein. 


\section{Graphical abstract}

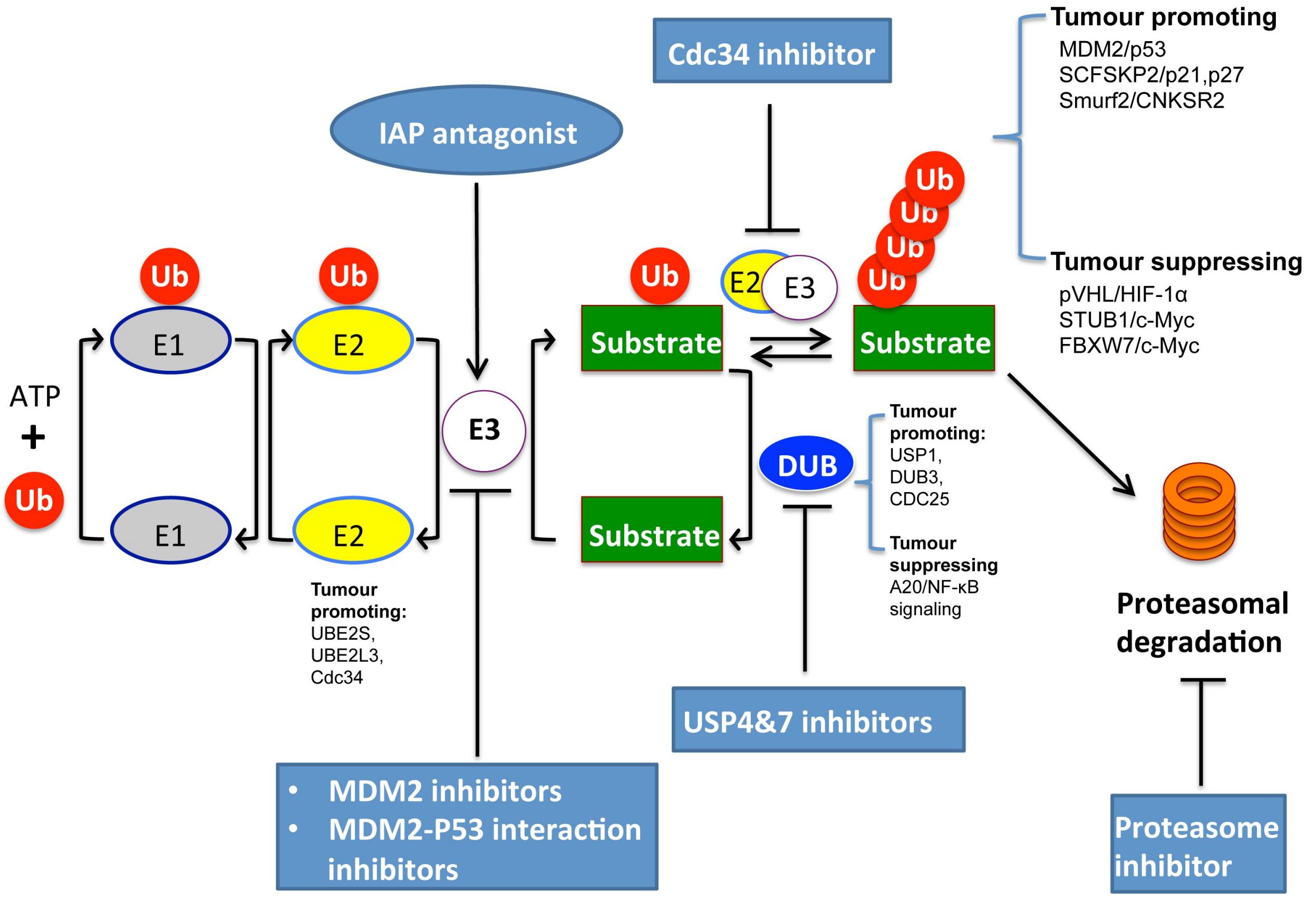




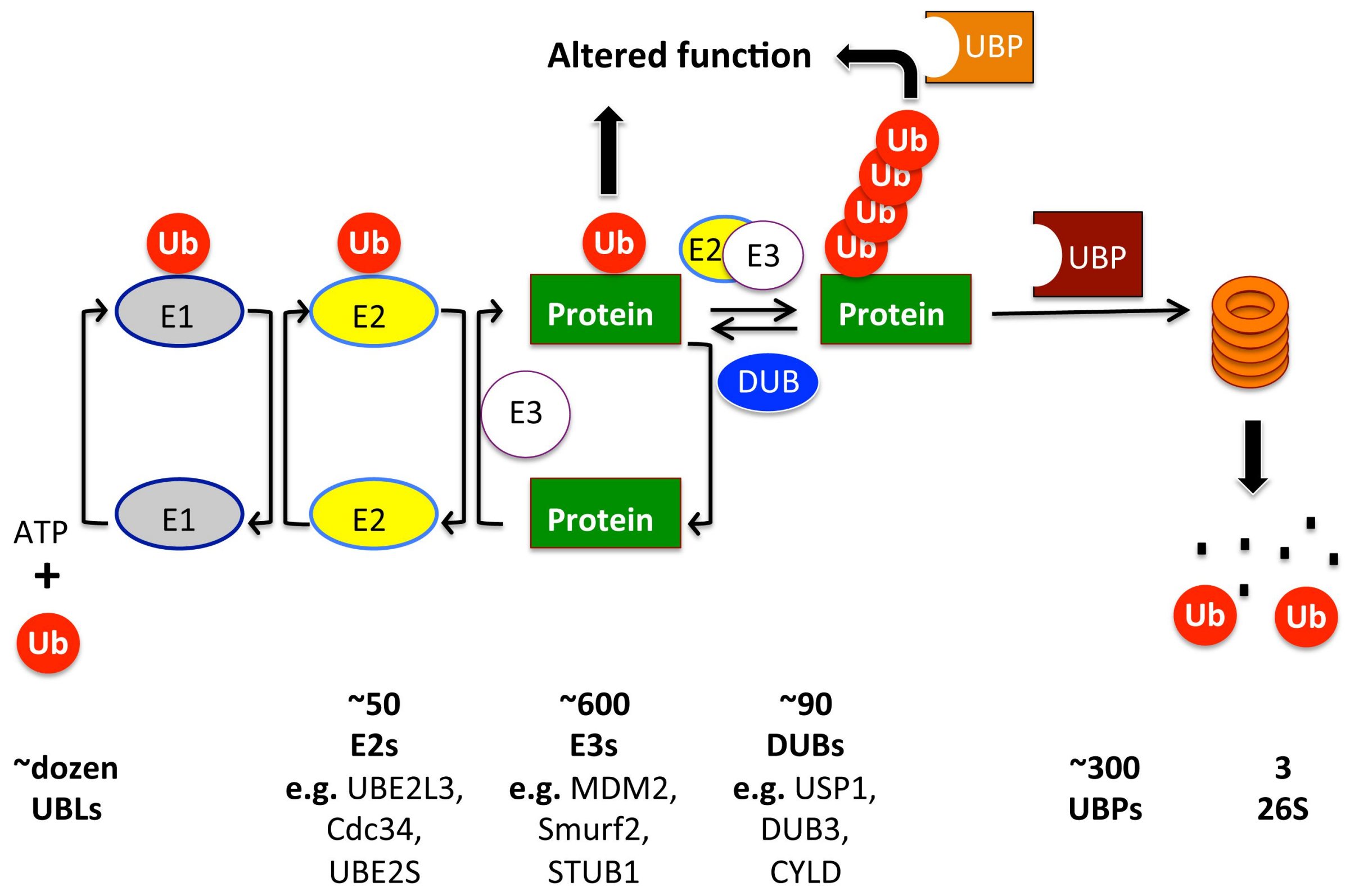




\section{Figure 2}

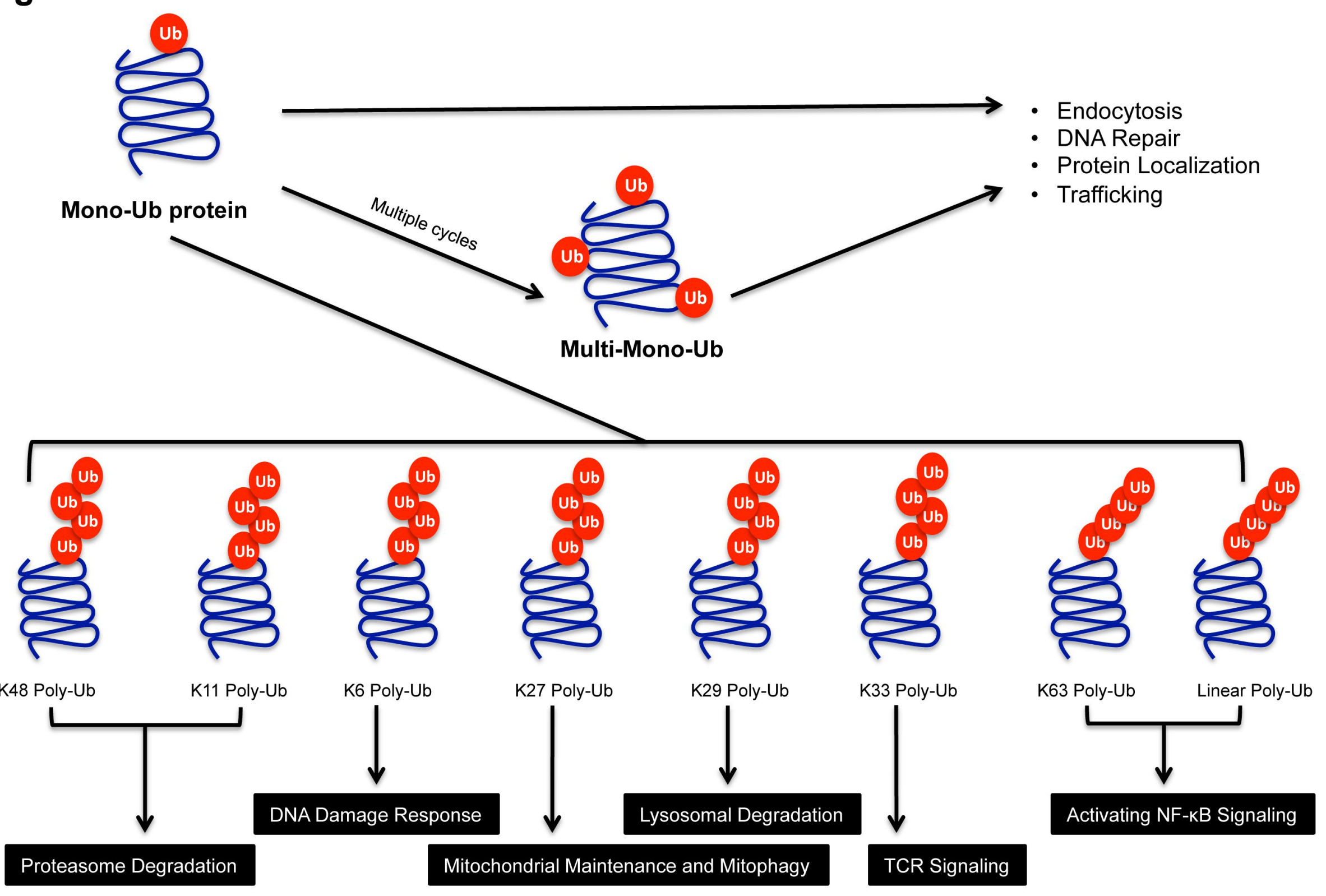




\section{Figure 3}

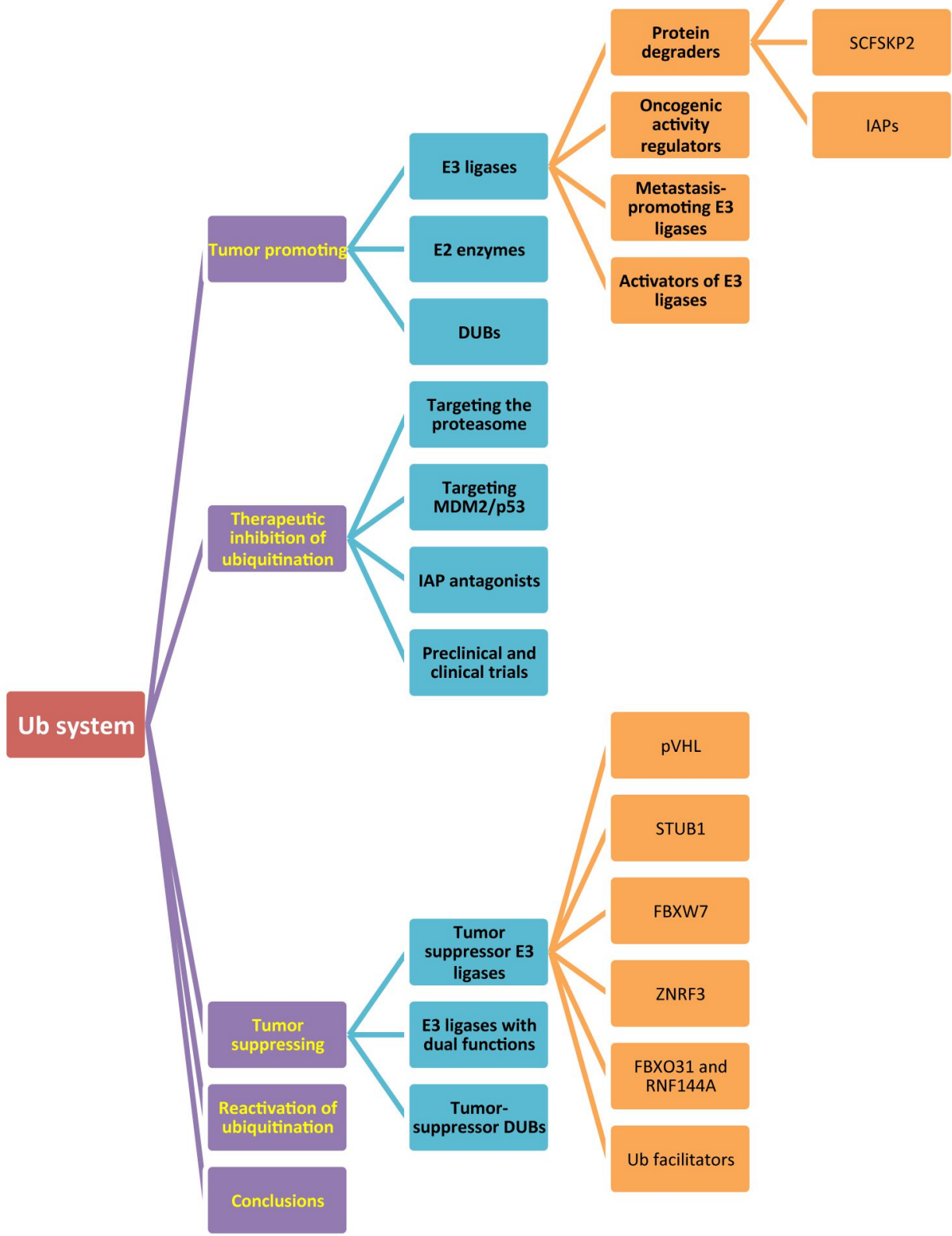


Figure 4

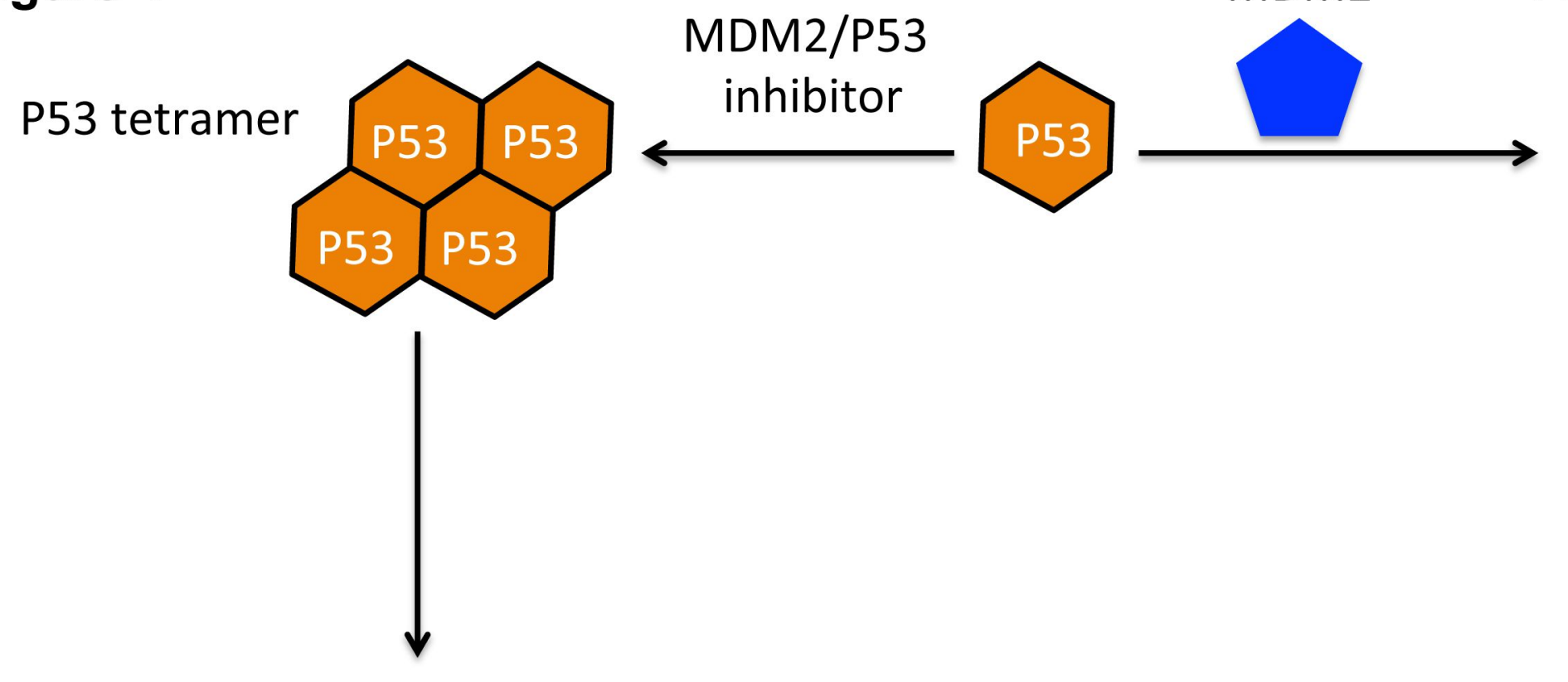

P53 ubiquitination

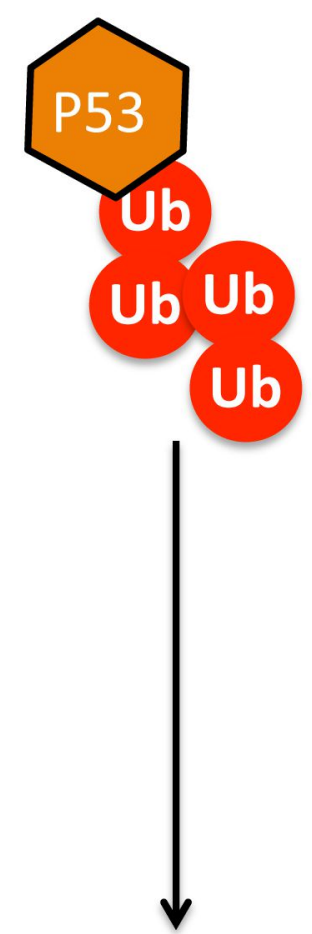

Ubiquitin-proteasome system

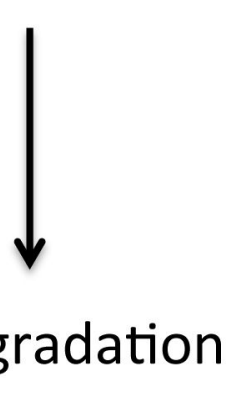

Degradation

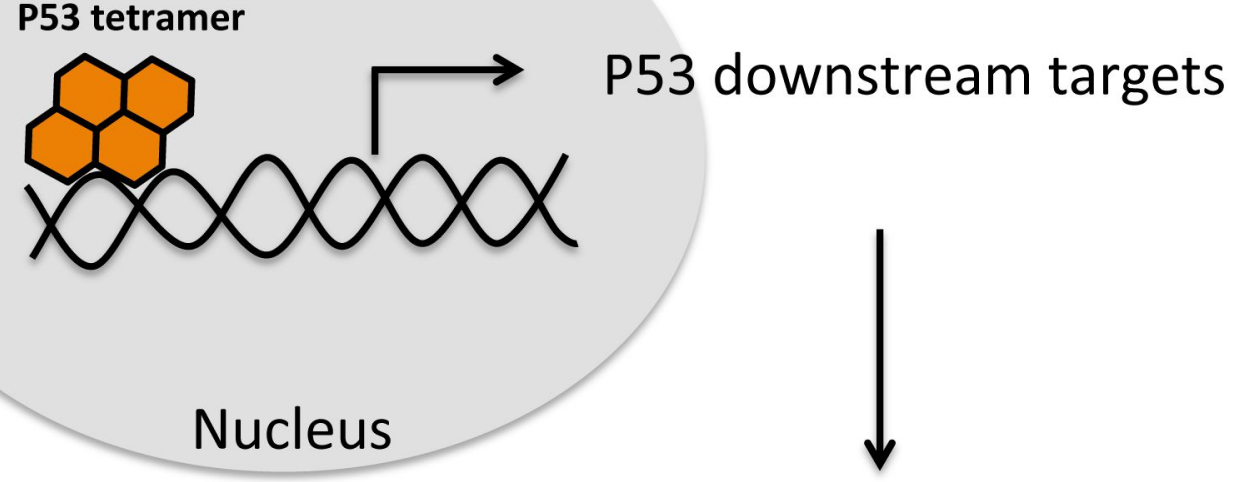

Tumor-suppressor activity 


\section{Figure 5}

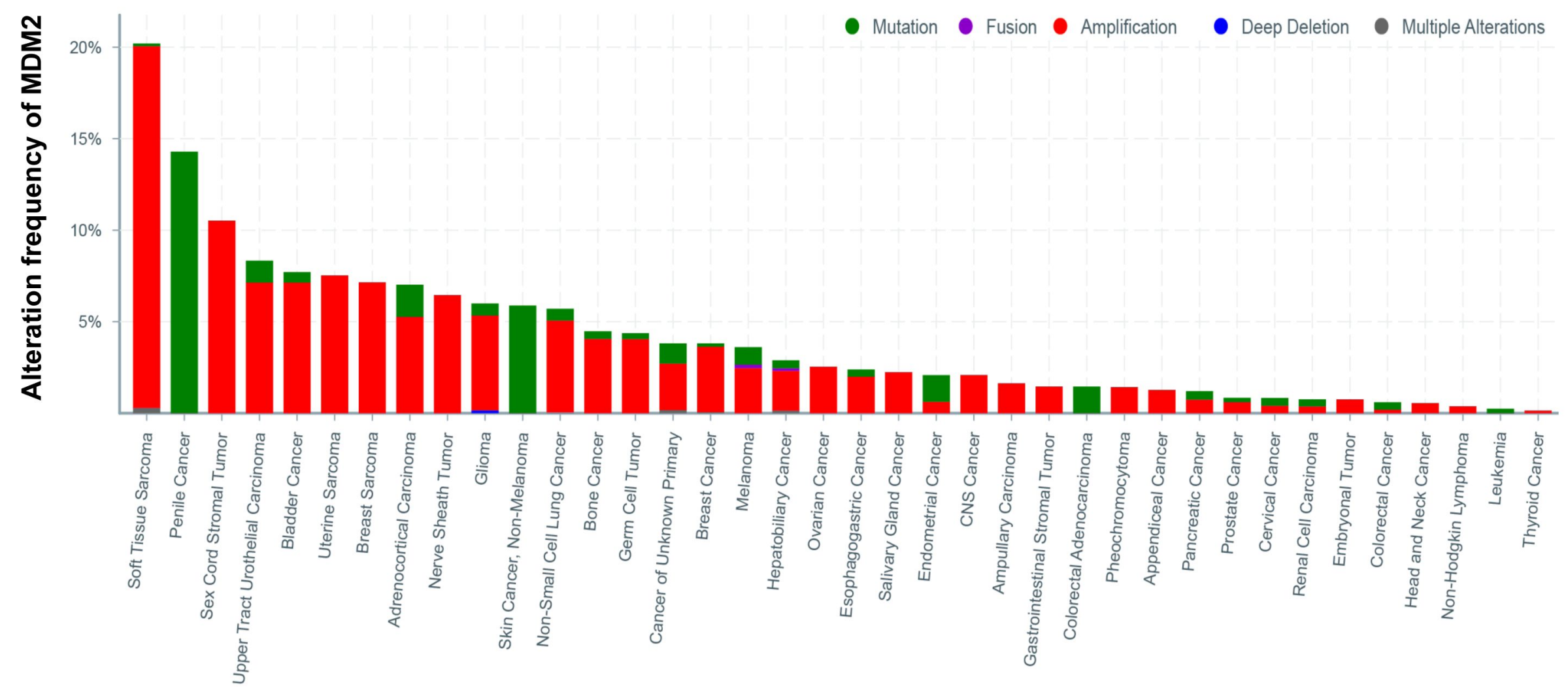


Figure 6

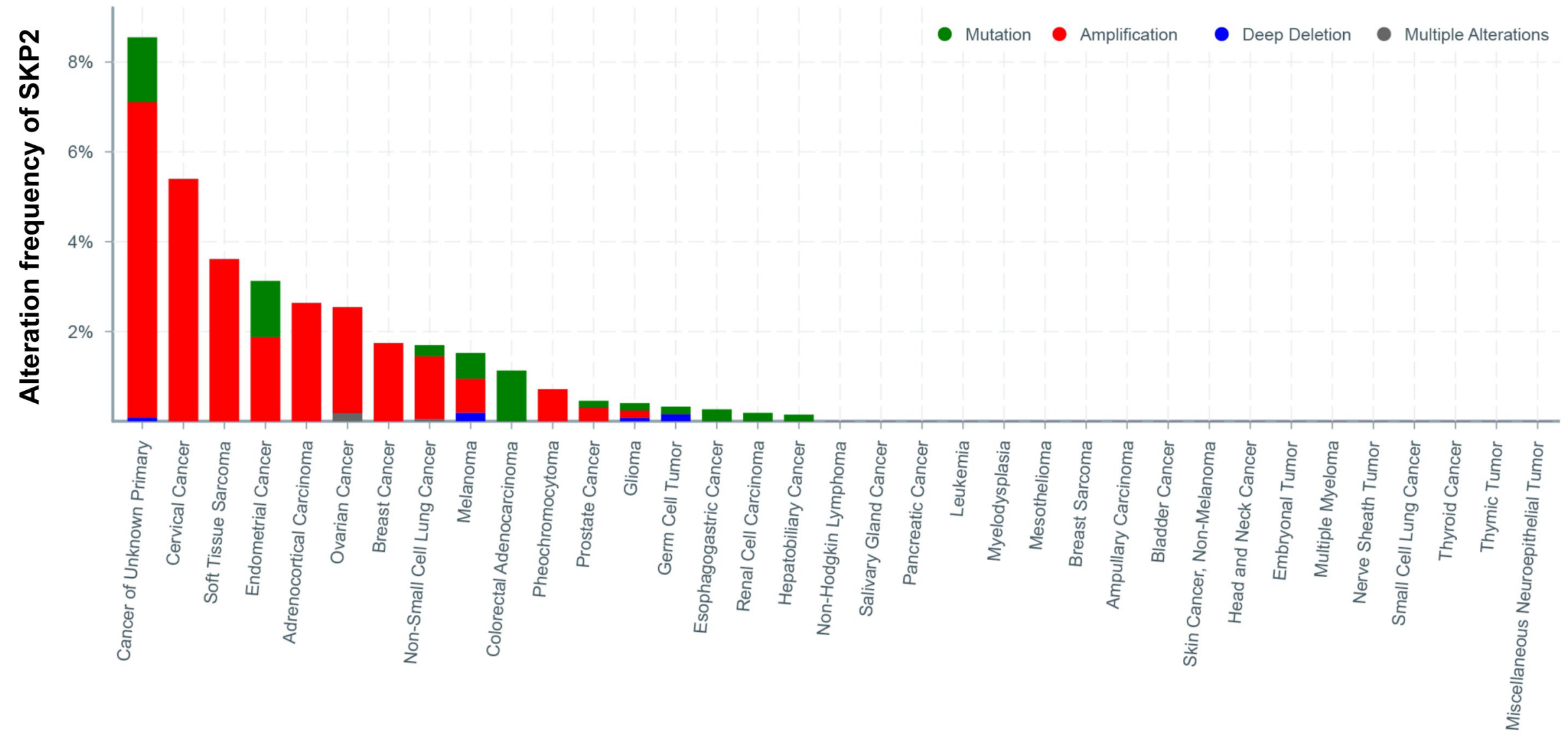


Figure 7

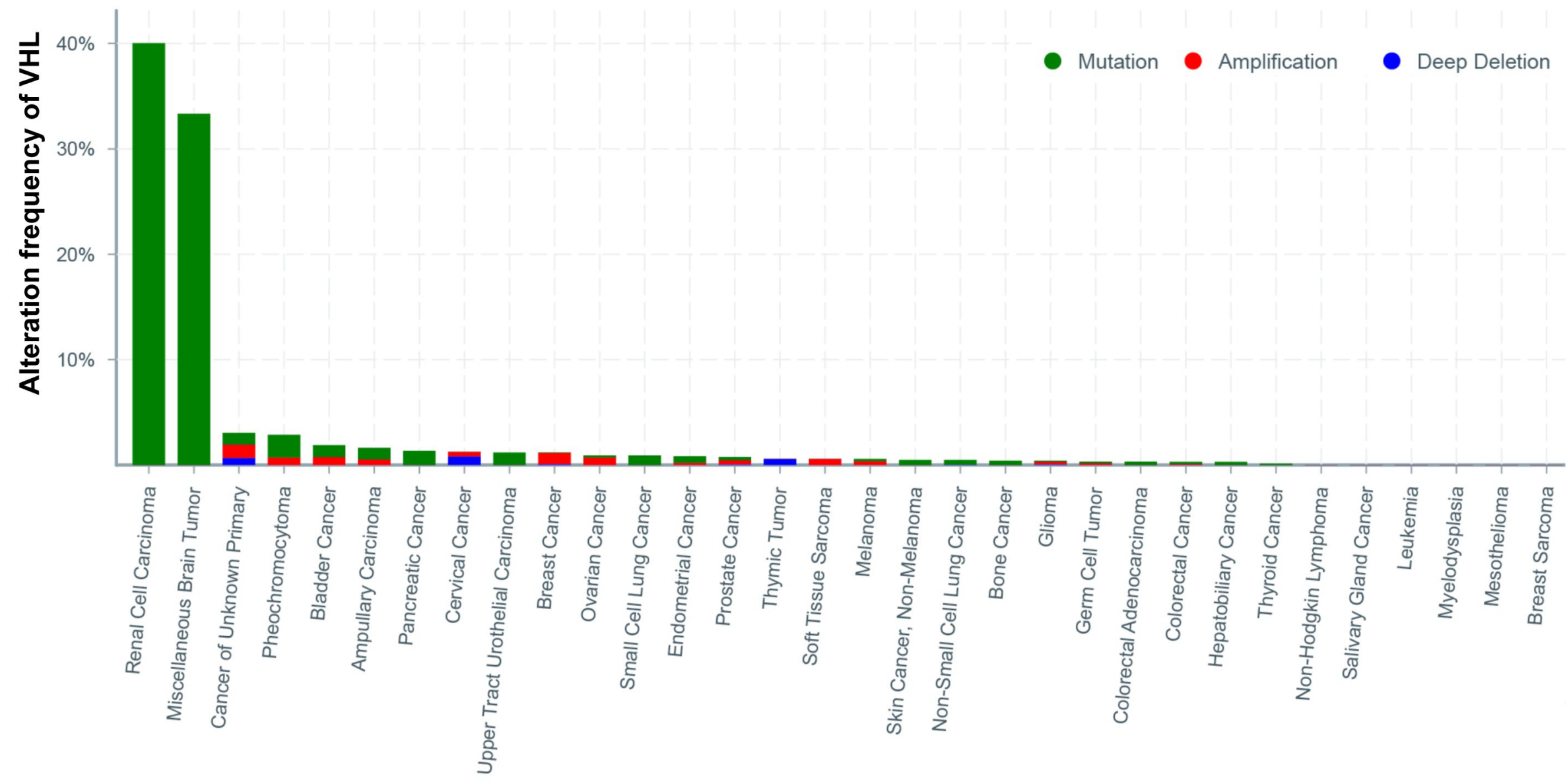




\section{Figure 8}
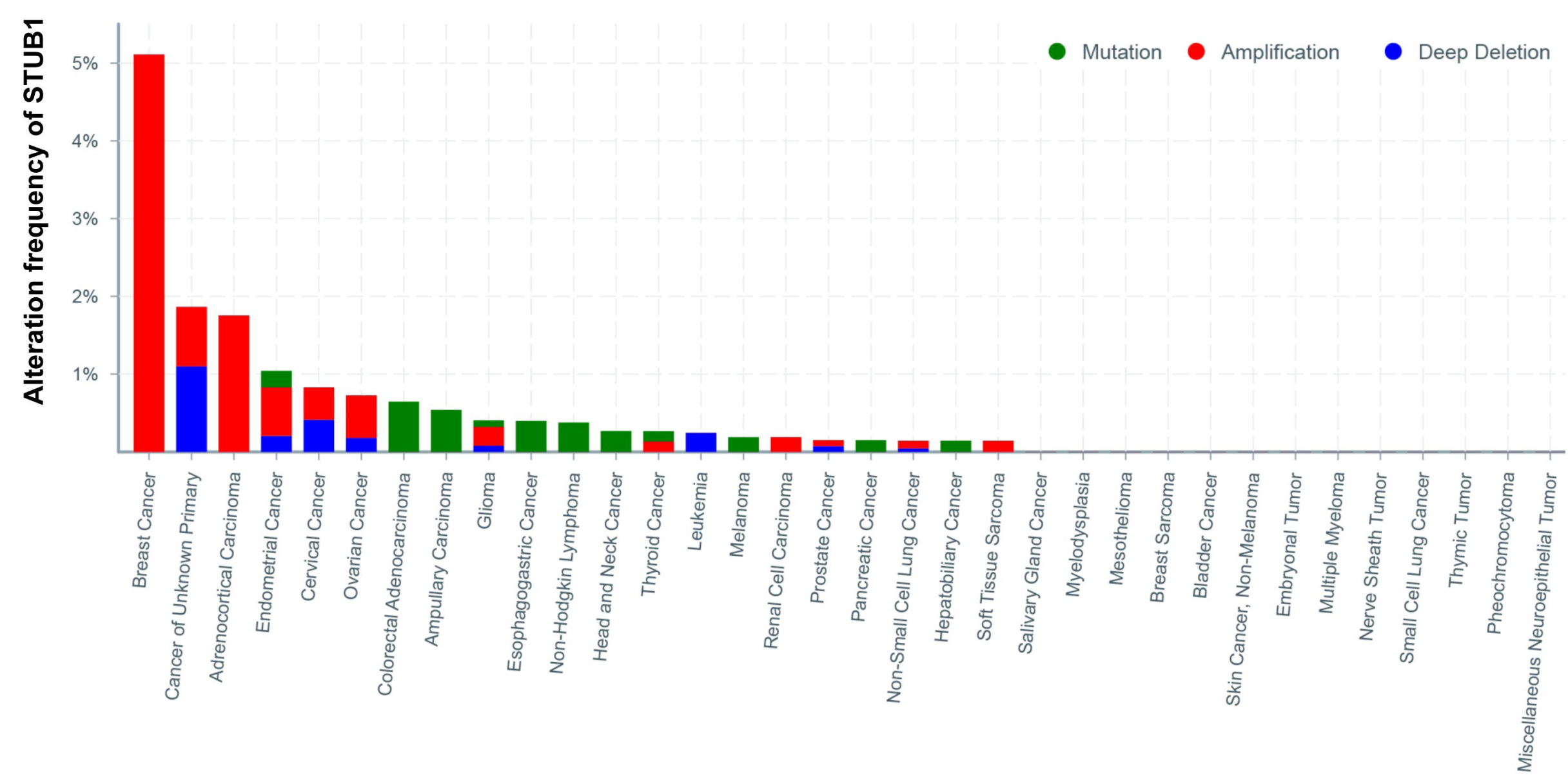


\section{Figure 9}

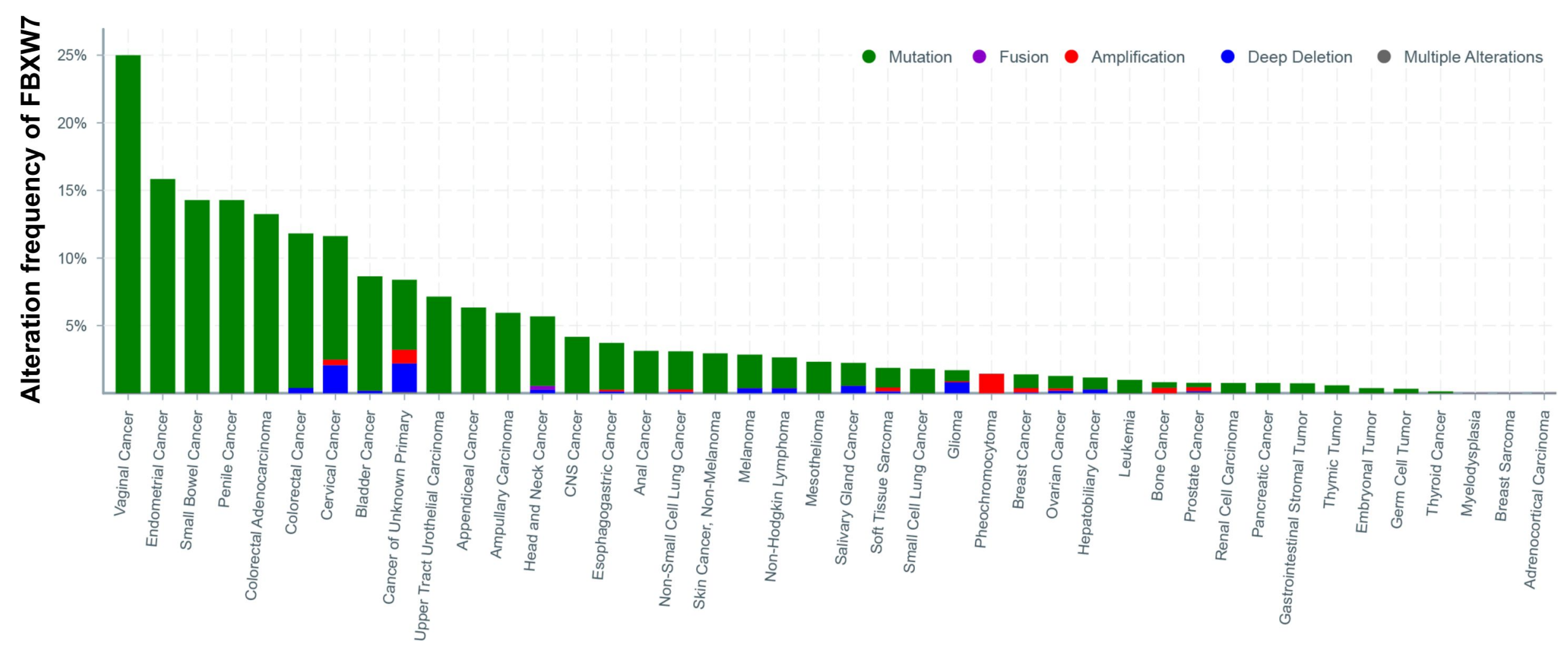




\section{Figure 11}

A
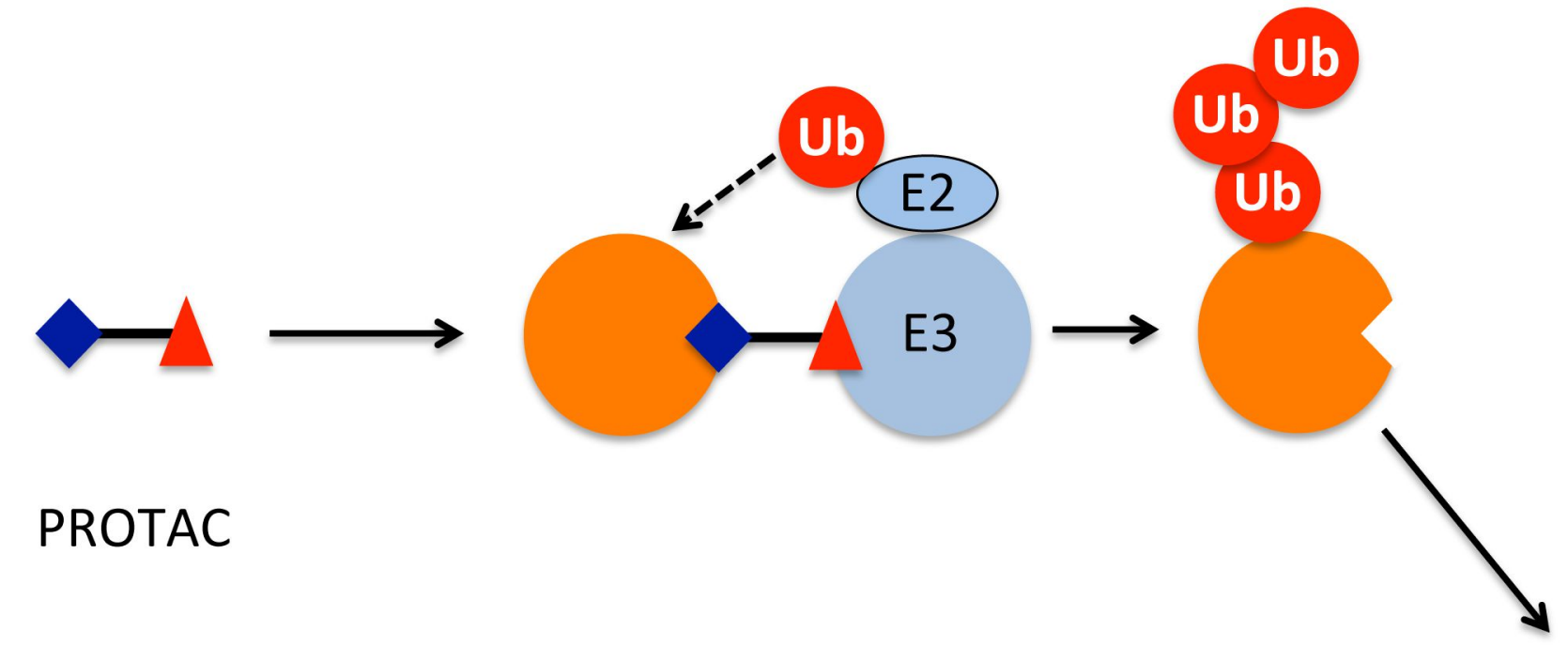

Target protein PROTAC

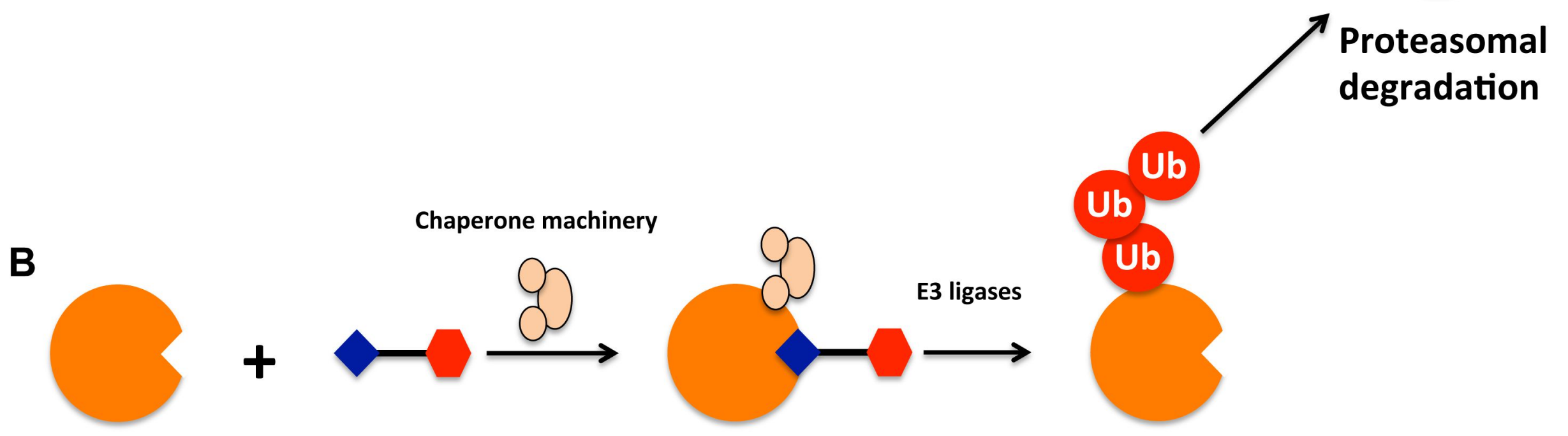

Target protein Hydrophobicity tag 